\title{
The XMM-Newton Optical Monitor survey of the Taurus molecular cloud ${ }^{\star, \star \star}$
}

\author{
M. Audard ${ }^{1, \star \star \star}$, K. R. Briggs ${ }^{2}$, N. Grosso ${ }^{3}$, M. Güdel ${ }^{2}$, L. Scelsi ${ }^{4}$, J. Bouvier $^{3}$, and A. Telleschi ${ }^{2}$ \\ ${ }^{1}$ Columbia Astrophysics Laboratory, Columbia University, 550 West 120th Street, Mail code 5247, New York, NY 10027, USA \\ e-mail: audard@astro.columbia.edu \\ 2 Paul Scherrer Institut, Würenlingen and Villigen, 5232 Villigen PSI, Switzerland \\ e-mail: [briggs; quedel; atellesc] aastro.phys.ethz.ch \\ ${ }^{3}$ Laboratoire d'Astrophysique de Grenoble, Université Joseph Fourier, 38041 Grenoble Cedex 9, France \\ e-mail: [Nicolas.Grosso; Jerome.Bouvier]@obs.ujf-grenoble.fr \\ 4 Dipartimento di Scienze Fisiche ed Astronomiche, Università di Palermo, Piazza del Parlamento 1, 90134 Palermo, Italy \\ e-mail: scelsi@astropa.unipa.it
}

Received 30 August 2006 / Accepted 8 November 2006

\section{ABSTRACT}

\begin{abstract}
Context. The Optical Monitor (OM) on-board XMM-Newton obtained optical/ultraviolet data for the XMM-Newton Extended Survey of the Taurus Molecular Cloud (XEST), simultaneously with the X-ray detectors.

Aims. With the XEST OM data, we study the optical and ultraviolet properties of TMC members, and to perform correlative studies between the X-ray and OM light curves. In particular, we aim to determine whether accretion plays a significant role in the optical/ultraviolet and X-ray emissions. The Neupert effect in stellar flares is also investigated.

Methods. Coordinates, average count rates and magnitudes were extracted from OM images, together with light curves with low time resolution (a few kiloseconds). For a few sources, OM FAST mode data were also available, and we extracted OM light curves with high time resolution. The OM data were correlated with Two Micron All Sky Survey (2MASS) data and with the XEST catalogue in the X-rays.

Results. The XEST OM catalogue contains 2148 entries of which 1893 have 2MASS counterparts. However, only 98 entries have $\mathrm{X}$-ray counterparts, of which 51 are known TMC members and 12 additional are TMC candidates. The OM data indicate that accreting stars are statistically brighter in the $U$ band than non-accreting stars after correction for extinction, and have $U$-band excesses, most likely due to accretion. The OM emission of accreting stars is variable, probably due to accretion spots, but it does not correlate with the X-ray light curve, suggesting that accretion does not contribute significantly to the X-ray emission of most accreting stars. In some cases, flares were detected in both X-ray and OM light curves and followed a Neupert effect pattern, in which the optical/ultraviolet emission precedes the X-ray emission of a flare, whereas the X-ray flux is proportional to the integral of the optical flux.
\end{abstract}

Key words. stars: coronae - stars: flare - stars: formation - stars: pre-main sequence - surveys - X-rays: stars

\section{Introduction}

The XMM-Newton Extended Survey of the Taurus Molecular Cloud (XEST) (Güdel et al. 2007a) is primarily focused on the $\mathrm{X}$-ray emission of young stellar and substellar objects (YSOs). However, since XMM-Newton (Jansen et al. 2001) is capable of observing simultaneously in the X-rays with the European Photon Imaging Cameras (Strüder et al. 2001; Turner et al. 2001) and the Reflection Grating Spectrometers (den Herder et al. 2001), and in the optical and ultraviolet regimes with the Optical Monitor (Mason et al. 2001), we have obtained deep images and

^ Appendix B is only available in electronic form at http: //www . aanda.org

$\star \star$ Appendix A is only available in electronic form at the CDS via anonymous ftp to cdsarc.u-strasbg.fr (130.79.128.5) or via http://cdsweb.u-strasbg.fr/cgi-bin/qcat?J/A+A/468/379

$\star \star \star$ New address (since September 2006): Integral Science Data Centre, Ch. d'Ecogia 16, 1290 Versoix, Switzerland \& Geneva Observatory, University of Geneva, Ch. des Maillettes 51, 1290 Sauverny, Switzerland; e-mail: Marc. Audard@obs . unige.ch sensitive light curves, primarily in the $U$ band, but also in the near-ultraviolet regime in some cases.

Simultaneous X-ray and optical/UV coverage of young stars provides the ideal means to study the physical processes occurring in the stellar upper atmospheres (e.g., Stelzer et al. 2003) or the interactions between an accretion disk and a star. For example, our understanding of the physics of flares in magnetically active stars can benefit from simultaneous monitoring. In the chromospheric evaporation model (e.g., Antonucci et al. 1984), magnetic reconnection in the corona injects accelerated particles propagating along the magnetic field lines, heating the chromosphere and the transition region through collisions (evident in emission in the optical/ultraviolet). Heated material (visible in soft X-rays) then moves up along the magnetic field lines into the corona where it cools. A "Neupert" effect (Neupert 1968) should, therefore, be observed in the optical/UV and Xray light curves, in which the time profile of the X-ray light curve is proportional to the time integral of the optical light curve (conversely, the optical flux has the same time profile as the derivative of the X-ray flux). This effect has been observed in 
main-sequence active stars (Hawley et al. 1995; Güdel et al. 2002a; Hawley et al. 2003; Güdel et al. 2004; Mitra-Kraev et al. 2005). Stellar Neupert effects were also reported from X-rays and radio gyrosynchrotron emission of accelerated particles gyrating along the magnetic fields (Güdel et al. 1996; Güdel et al. 2002b; Smith et al. 2005), although not all multi-wavelength observations necessarily detect typical Neupert effects (e.g., Stelzer et al. 2003; Osten et al. 2005), probably because of different conditions in the flaring source.

Optical studies of young stars have also provided evidence of rotational modulation of active regions, suggesting that a solartype magnetic activity operates in T Tauri stars (e.g., Rydgren \& Vrba 1983; Rydgren et al. 1984; Bouvier et al. 1988; Bouvier \& Bertout 1989; Bouvier 1990; Bouvier et al. 1993; Vrba et al. 1993; Bouvier et al. 1995, 1997, etc.). Detailed analyses indicate that weak-line T Tauri stars (WTTS) on average rotate faster than the still accreting classical T Tau stars (CTTS), possibly due to disk locking or loss of angular momentum due to stellar winds in CTTS (Bouvier et al. 1993). In addition, rotational modulation in some CTTS can be dominated by hot spots due to accreting material falling from the accretion disk onto the stellar surface (Vrba et al. 1986; Bouvier \& Bertout 1989; Vrba et al. 1989; Bouvier et al. 1993; Herbst et al. 1994; Bouvier et al. 1995).

Although most low-mass accreting stars display X-ray spectra typical of hot coronal plasma, at least a few of them display either lower temperature spectra or densities atypical of a stellar corona, or both, indicating that accretion could play a significant role in the production of X-rays (Kastner et al. 2002; Stelzer \& Schmitt 2004; Schmitt et al. 2005; Ness \& Schmitt 2005; Robrade \& Schmitt 2006; Telleschi et al. 2007a). In some cases, the soft X-ray component could arise from shocks in jets originating from the young accreting stars (Güdel et al. 2005; Kastner et al. 2005; Güdel et al. 2007b). In low-mass young stars with optical outbursts, the sudden rise in mass accretion rate also appears to impact on the X-ray emission. During the accretion outburst of V1647 Ori, the mean X-ray flux closely tracked the near-infrared luminosity, and the X-ray spectrum hardened (Kastner et al. 2004; Grosso et al. 2005; Kastner et al. 2006). On the other hand, Audard et al. (2005) observed little X-ray flux variability in the early epochs of the outburst, in contrast to the optical/near-infrared flux enhancements, but they found a change in the X-ray spectrum of the young star V1118 Ori from a dominant hot plasma pre-outburst to a cool plasma during the outburst. The coronal hot plasma essentially disappeared, probably because the inner accretion disk disrupted the coronal loops. Finally, accretion may also be a dominant mechanism in the more massive, accreting Herbig Ae stars (Swartz et al. 2005), although magnetically confined winds may be a good alternative as well (Telleschi et al. 2007b).

Optical and X-ray correlations of young stars can, therefore, provide important information on flare physics and rotational modulation due to spots and active regions in magnetically active stars (e.g., Flaccomio et al. 2005) or due to hot accretion spots. Multi-wavelength studies are ideal to determine to what extent accretion plays a significant role in the production of X-rays in young accreting stars. Recently, Stassun et al. (2006) studied the correlation between the optical $(B V R I)$ and X-ray light curves of young stars in the Orion Nebula Cluster. The optical observations covered the 13-day Chandra observation of the cluster, and the typical exposure times ranged from $5 \mathrm{~s}$ for short exposures to 420-720 s for long exposures. However, the observing cadence was about 1 per hour for each filter. Stassun et al. (2006) found little evidence of correlations between the optical and X-ray variability, although there were some exceptions.
In this paper, we report on the Optical Monitor (OM) data obtained as part of the XEST. Section 2 describes the OM data, whereas we introduce the OM catalogue in Sect. 3. The vast majority of our optical (and UV in a few cases) detections is of sources which are probably foreground or background sources. We provide the full OM catalogue of detected sources as online material, although this paper focuses specifically on known or probable TMC members (including new membership candidates identified by Scelsi et al. 2007). A separate paper focuses on the OM survey of brown dwarfs (Grosso et al. 2007a). Section 4 makes use of the OM data and data compiled by Güdel et al. (2007a) in order to derive basic properties of the OM TMC sample. Since the OM and X-ray detectors observed simultaneously, we also study correlations between the optical/ultraviolet and X-ray light curves for TMC members in Sect. 5.

\section{Optical Monitor data}

The Optical Monitor (OM; Mason et al. 2001) is a 30-cm optical/UV telescope that can provide coverage in the optical and UV regimes (bandwidth $180-600 \mathrm{~nm}$ ) simultaneously with the $\mathrm{X}$-ray cameras. The OM detector is a micro-channel plate (MCP) intensified CCD. The final array has a format of $2048 \times 2048$ pixels, each pixel having a size of about $0{ }^{\prime} 48$, leading to a square field-of-view (FOV) of about $17^{\prime} \times 17^{\prime}$, which covers the central part of the X-ray cameras (15'-radius FOV). The OM carries a wheel of filters $(V, U, B, U V W 1, U V M 2, U V W 2$, and a broad white light filter) and 2 grisms for the visible and UV ranges. In this paper, we report on OM data taken with the $U$ $(\approx 300-400 \mathrm{~nm})$ and $U V W 2(\approx 175-250 \mathrm{~nm})$ filters. The OM point-spread-function varies from $1^{\prime \prime} .4$ to $2^{\prime \prime}$, depending on the filter. The OM can operate in the "Imaging" or "Fast" modes; of particular interest here, in the default Imaging mode ("Image" in Table 1), a set of 5 consecutive exposures is taken, each covering a different portion of the FOV. In each of the 5 exposures, a large window $\left(W_{1, \ldots, 5}\right.$; with $1^{\prime \prime}$ pixels) is complemented by a smaller central imaging mode window of size $2^{\prime} \times 2^{\prime}$ with 0 '. $^{\prime} 5$ pixel resolution $\left(W_{0}\right)$. Thus, the sequence is exposure $1: W_{0}+W_{1}$, exposure 2: $W_{0}+W_{2}$, etc. until exposure 5: $W_{0}+W_{5}$, after which the sequence can start again. An example of the configuration can be found in the XMM-Newton Users' Handbook (Ehle et al. 2005). In the default Fast mode ("Image Fast" in Table 1), a similar set of images is obtained together with an additional central window $(\mathrm{CW} ; 22 \times 23$ pixels, i.e., $10.5 \times 10.5)$ that is operated in fast mode with a time resolution of $0.5 \mathrm{~s}$. Consequently, the sequence of images is exposure 1: $W_{0}+W_{1}+\mathrm{CW}$, exposure 2: $W_{0}+W_{2}+\mathrm{CW}$, etc. until exposure 5: $W_{0}+W_{5}+\mathrm{CW}$. This means that the on-axis target should be monitored continuously with high time resolution, and 5 times in the small central $2^{\prime} \times 2^{\prime} W_{0}$ imaging window (with each an exposure equal to the integration time of the exposure), and once in the large, central $1^{\prime \prime}$-pixel window. If a secondary target is located in a sky area covered by the small central imaging window $W_{0}$, it is observed in a similar fashion as above, except that no fast mode data is available. If it is located in an area outside the $2^{\prime} \times 2^{\prime}$ window, it will be observed only in one of the 5 consecutive large imaging window, i.e., once per OM exposure. Clearly, the best time coverage for a secondary target is when it is located in a sky area covered by the small central window. Note that other imaging modes can be used as well. For example, in "Full-Frame" imaging mode, images of the whole OM FOV can be obtained either in full resolution ( 0 '5 pixel size; "High Resolution") or in low ( 1 " pixel size; "Low Resolution"). Additional user-specified mode can be used as well (see XEST-28 in Table 1). Because the OM FOV 
Table 1. OM-specific observation Log.

\begin{tabular}{|c|c|c|c|c|c|c|c|c|}
\hline 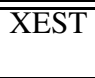 & 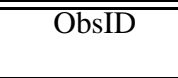 & $\begin{array}{l}\mathrm{RA}^{a} \\
\mathrm{~h} \mathrm{~m} \mathrm{~s}\end{array}$ & $\begin{array}{l}\delta^{a} \\
\circ{ }^{a}\end{array}$ & $\begin{array}{l}\mathrm{PA}^{a} \\
\left({ }^{\circ}\right)\end{array}$ & $\begin{array}{c}\mathrm{OM} \\
\text { Filter }\end{array}$ & $\begin{array}{c}\text { OM } \\
\text { Mode }\end{array}$ & $\begin{array}{l}\text { Fast } \\
\text { Data }\end{array}$ & $\begin{array}{l}\text { Image } \\
\text { Exposures }\end{array}$ \\
\hline 01 & 0301500101 & 042159.4 & +193206 & 80.01 & $U V W 1$ & Image Fast & $\bar{Y}$ & $18 \times 3540 \mathrm{~s}$ \\
\hline 02 & 0203540201 & 042719.6 & +260925 & 82.05 & $U$ & Full-Frame Low Res ${ }^{b}$ & $\mathrm{~N}$ & $4 \times 5000 \mathrm{~s}, 3170 \mathrm{~s}$ \\
\hline 03 & 0203540301 & 043218.9 & +242228 & 81.92 & $U$ & Image Fast & $\mathrm{Y}$ & $20 \times 1500 s$ \\
\hline 04 & 0203540401 & 043334.4 & +242108 & 261.88 & $U$ & Image Fast & $\mathrm{Y}$ & $12 \times 1640 s$ \\
\hline 05 & 0203540501 & 043934.9 & +254146 & 262.68 & $U$ & Full-Frame Low $\operatorname{Res}^{b}$ & $\mathrm{~N}$ & $4000 \mathrm{~s}, 4479 \mathrm{~s}, 2 \times 5000 \mathrm{~s}$ \\
\hline 06 & 0203540601 & 040442.9 & +261856 & 79.03 & $U$ & Full-Frame Low Res ${ }^{b}$ & $\mathrm{~N}$ & $4 \times 5000 \mathrm{~s}$ \\
\hline 07 & 0203540701 & 044112.5 & +254637 & 262.61 & $U$ & Full-Frame Low $\operatorname{Res}^{b}$ & $\mathrm{~N}$ & $3 \times 5000 \mathrm{~s}, 1679 \mathrm{~s}$ \\
\hline 08 & 0203540801 & 043552.9 & +225423 & 81.90 & $U$ & Image Fast & $\mathrm{Y}$ & $10 \times 1260 s, 10 \times 1240 s$ \\
\hline 09 & 0203540901 & 043555.1 & +223924 & 261.85 & $U$ & Full-Frame Low Res ${ }^{b}$ & $\mathrm{~N}$ & $5 \times 5000 \mathrm{~s}$ \\
\hline 10 & 0203542201 & 044220.9 & +252035 & 262.27 & $U$ & Image Fast & $\mathrm{Y}$ & $15 \times 1780 s$ \\
\hline 11 & 0203541101 & 042151.1 & +265733 & 81.69 & $U$ & Full-Frame Low Res ${ }^{b}$ & $\mathrm{~N}$ & $5 \times 5000 \mathrm{~s}$ \\
\hline 12 & 0203542101 & 043517.4 & +241500 & 261.59 & $U$ & Image Fast & $\mathrm{Y}$ & $15 \times 1500 \mathrm{~s}$ \\
\hline 13 & 0203541301 & 042952.0 & +243647 & 81.55 & $U$ & Full-Frame Low Res ${ }^{b}$ & $\mathrm{~N}$ & $3 \times 5000 s, 1679 s$ \\
\hline 14 & 0203541401 & 043030.6 & +260214 & 262.72 & $U$ & Full-Frame Low $\operatorname{Res}^{b}$ & $\mathrm{~N}$ & $2 \times 5000 s, 4179 s$ \\
\hline 15 & 0203541501 & 042942.4 & +263251 & 262.81 & $U$ & Full-Frame Low Res ${ }^{b}$ & Y & $12 \times 1260 s, 5 \times 1380 s$ \\
\hline 16 & 0203541601 & 041943.0 & +271334 & - & - & - & - & - \\
\hline 17 & 0203541701 & 043321.2 & +225241 & 261.86 & $U$ & Full-Frame Low Res ${ }^{b}$ & $\mathrm{~N}$ & $3 \times 4170 s$ \\
\hline 18 & 0203541801 & 043354.7 & +261328 & 83.07 & $U$ & Image Fast & $\mathrm{Y}$ & $5 \times 1360 \mathrm{~s}, 5 \times 1260 \mathrm{~s}, 10 \times 1200 \mathrm{~s}$ \\
\hline 19 & 0203541901 & 043243.0 & +255232 & 82.83 & $U$ & Image Fast & Y & $15 \times 1680 s, 5 \times 1660 s$ \\
\hline 20 & 0203542001 & 041412.9 & +281212 & 78.06 & $U$ & Image Fast & $\mathrm{Y}$ & $10 \times 2480 s$ \\
\hline 21 & 0101440701 & 042159.0 & +281808 & - & - & - & - & - \\
\hline 22 & 0109060301 & 043139.0 & +181000 & 83.58 & $U V W 2$ & Image $^{c}$ & $\mathrm{~N}$ & $5 \times 1000 s$ \\
\hline 23 & 0086360301 & 041831.2 & +282716 & 259.74 & $U V W 2$ & Image & $\mathrm{N}$ & $5 \times 1000 s$ \\
\hline 24 & 0086360401 & 041831.2 & +282716 & 259.67 & $U V W 2$ & Image & $\mathrm{N}$ & $5 \times 1000 \mathrm{~s}$ \\
\hline 25 & $0152680201^{d}$ & 043455.5 & +242854 & 244.71 & $U V W 2$ & Image & $\mathrm{N}$ & $10 \times 980 \mathrm{~s}$ \\
\hline 26 & 0101440801 & 045559.0 & +30 3402 & - & - & - & - & - \\
\hline 27 & 0201550201 & 035407.9 & +315301 & - & - & - & - & - \\
\hline 28 & 0200370101 & 041915.8 & +290627 & 82.13 & $U V W 1$ & Science User Defined ${ }^{c, e}$ & $\mathrm{Y}$ & $25 \times 4000 \mathrm{~s}, 2 \times 2200 \mathrm{~s}$ \\
\hline
\end{tabular}

${ }^{a}$ Nominal boresight coordinates (J2000.0) and average spacecraft position angle. ${ }^{b} 1024 \times 1024$ pixel images with $1^{\prime \prime}$ pixel size $\left(17^{\prime} \times 17^{\prime}\right) .{ }^{c} \mathrm{Grism}$ exposures taken after the imaging exposures. ${ }^{d}$ This observation is part of a campaign on AA Tau that will be presented extensively elsewhere. ${ }^{e} 624 \times 624$ pixel images with $0{ }^{\prime} 5$ pixel size $\left(5^{\prime} \times 5^{\prime}\right)$ together with a $10,5 \times 10^{\prime}, 5$ window in Fast mode.

is smaller $\left(17^{\prime} \times 17^{\prime}\right)$ than the EPIC field-of-view $\left(15^{\prime}\right.$ radius), several interesting X-ray sources did not fall on the OM detector, and, therefore, no photometry or light curve could be obtained.

The OM data were reduced with the XMM-Newton Science Analysis System (SAS) 6.1. We have processed the "Imaging" and "Fast" mode data using the metatasks omichain and omfchain, respectively, with default parameters, except for the omdetectminsignificance parameter of omichain, which determines the minimum significance of a source to be included in the source-list file and which we changed from 1.0 to 3.0. In Table 1, we provide an observation log of the XEST fields (Güdel et al. 2007a), with an emphasis on the OM-specific parameters. In particular, we provide the imaging mode and emphasize when a Fast mode window was available, and we provide the exposures of the individual imaging windows. For example, for XEST-01, for the first exposure, the imaging windows $W_{0}$ and $W_{1}$ observed simultaneously for $3540 \mathrm{~s}$, then (after some overhead delay), $W_{0}$ and $W_{2}$ observed for another $3540 \mathrm{~s}$, etc. Since the XEST-01 OM data were obtained in FAST mode as well, the $\mathrm{CW}$ window was operating in parallel. For XEST-02, $W_{0}+W_{1}, W_{0}+W_{2}, W_{0}+W_{3}$, $W_{0}+W_{4}$ each observed for $5000 \mathrm{~S}$, while the last exposure with windows $W_{0}$ and $W_{5}$ observed for 3170 s only.

For "Imaging" mode data, we obtained images and source lists for individual exposures, a mosaicked image, and a merged source list. Each individual source list included, in particular, the exposure source number, sky and galactic coordinates, raw and corrected count rates, magnitudes, quality flags, and the source identification in the final source list. Consequently, it was possible, knowing the source identification, to obtain exposurespecific count rates and magnitudes, i.e., low time-resolution light curves ${ }^{1}$. However, SAS 6.1 identified a significant number of spurious sources, mostly in the ghost image near a bright star produced by internal reflection of light within the detector window, in the enhanced "ring" of emission near the center of the detector (due to the reflection of diffuse sky light from outside the FOV), and in fixed pattern noise around bright sources (Ehle et al. 2005). We manually inspected the mosaicked image, merged source list identifications, and removed obvious misidentifications. We also modified the merged source list to ensure that the same source had the same source identification in each exposure; indeed, the omsrclistcomb task did not combine the source lists accurately in a few cases, i.e., mostly bright sources for which strong fixed pattern noise introduced small positional inaccuracies that were not properly identified by SAS 6.1. We note that SAS 6.5 eliminates most of the above issues; however, this version of the SAS incorrectly combines the source lists, and, therefore, we preferred not to use it. Figure 1 shows an example of an OM image with some artefacts.

For "Fast" mode data, in addition to individual images, we obtain event lists and time series files with high time resolution (the default value is $10 \mathrm{~s}$ with omfchain) for each exposure. We merged the different time series files into a single time series file for further analysis.

\footnotetext{
${ }^{1}$ For point sources identified in both the small $0.5 \times 0 .{ }^{\prime} 5$ and the larger $1^{\prime \prime} \times 1^{\prime \prime}$ central windows, we used count rates and magnitudes from the small window because of the smaller pixel bin size and of the more frequent time sampling.
} 


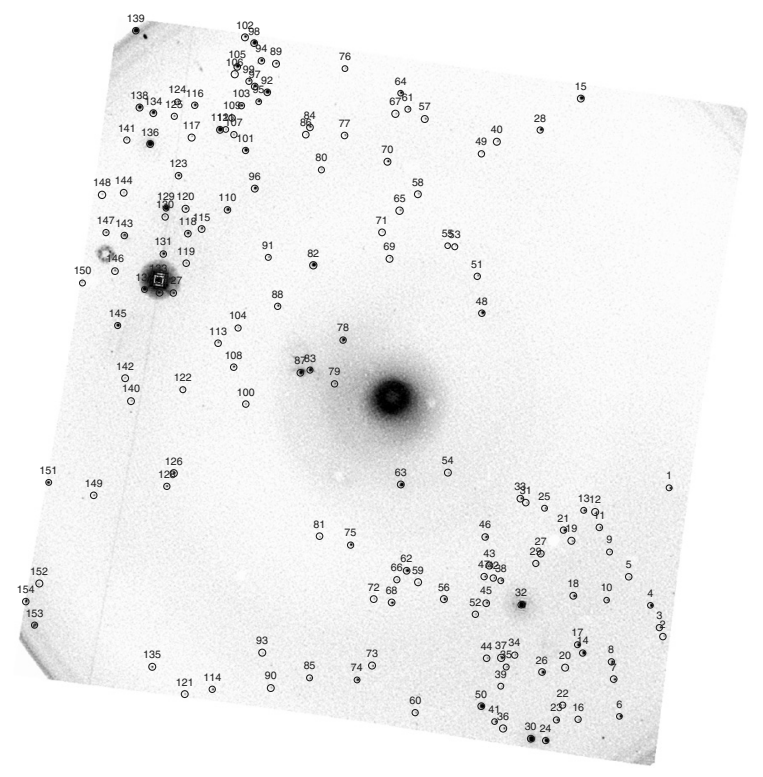

Fig. 1. Example of an OM image. This rich field (XEST-11, toward FS Tau) shows artefacts, such as the "ring" of emission at the center (due to the reflection of diffuse sky light from outside the FOV), fixed pattern noise around the bright source XEST-11-OM-133 (HD 283579, which is not classified as a TMC member), and a ghost ring below source XEST-11-OM-147 which is also caused by XEST-11-OM-133. Other artefacts can be seen (trail along the bright source, defects on the detector)

Table 2. OM astrometric correction vectors in arcseconds.

\begin{tabular}{crrl}
\hline \hline XEST & \multicolumn{1}{c}{$\Delta X$} & \multicolumn{1}{c}{$\Delta Y$} & rms \\
\hline 01 & 3.086 & -2.462 & 0.90 \\
02 & 4.338 & -1.016 & 0.62 \\
03 & 3.320 & -0.970 & 0.52 \\
04 & -1.471 & 3.632 & 0.83 \\
05 & -3.561 & 4.579 & 0.52 \\
06 & 3.948 & -3.227 & 0.57 \\
07 & -3.240 & 4.412 & 0.99 \\
08 & 2.955 & -0.800 & 0.81 \\
09 & -2.728 & 5.588 & 0.62 \\
10 & -2.219 & 2.623 & 0.61 \\
11 & 3.847 & -0.137 & 0.61 \\
12 & -2.675 & 3.093 & 0.61 \\
13 & 2.143 & -1.162 & 0.86 \\
14 & -4.529 & 3.239 & 0.68 \\
15 & -3.891 & 5.163 & 0.71 \\
17 & -3.824 & 5.114 & 0.58 \\
18 & 2.893 & -1.391 & 0.92 \\
19 & 3.617 & -0.044 & 0.62 \\
20 & 6.201 & -5.232 & 0.48 \\
22 & 1.586 & -1.008 & 0.06 \\
23 & 0.368 & 1.105 & $0.00^{a}$ \\
24 & -1.363 & 0.720 & $0.00^{a}$ \\
25 & -3.065 & 4.090 & 1.63 \\
28 & 5.519 & -0.994 & 0.77 \\
\hline
\end{tabular}

${ }^{a}$ Only V410 Tau detected in the OM.

\section{Optical Monitor catalogue}

A catalogue of OM sources was compiled, cross-checked with the Two Micron All Sky Survey (2MASS) and the XEST X-ray sources. We provide average photometry and source coordinates as compiled in the combined source lists in the imaging mode data. We calculated boresight corrections to OM coordinates by cross-correlating with the 2MASS catalogue (search radius of $3^{\prime \prime}$ ) and by means of iterative steps until the median position offsets became zero. Table 2 provides the position offsets $(\Delta X, \Delta Y)$ in arcseconds $\left(\mathrm{RA}_{\text {corr }}=\mathrm{RA}_{\mathrm{OM}}+\Delta X / \cos \left(\delta_{2 \mathrm{MASS}}\right)\right.$ and $\left.\delta_{\text {corr }}=\delta_{\mathrm{OM}}+\Delta Y\right)$. We also cross-correlated the $\mathrm{OM}$ and the $\mathrm{X}$-ray catalogues (Güdel et al. 2007a) using 2MASS-corrected coordinates (search radius of $3^{\prime \prime}$ ). Minor manual interventions were necessary when two close sources were separated in the OM but not in X-rays (UZ Tau E+W(AB), HP Tau/G2 and HP $\mathrm{Tau} / \mathrm{G} 3 \mathrm{AB})$. We used the same detection parameters for all observations. Consequently, the source detection process is not optimized for each exposure; however, a visual inspection showed that the chosen parameters were generally sufficient, although we do not claim completeness in the XEST OM catalogue.

Table 3 provides the first 30 entries of the full OM catalogue, in increasing right ascension. In Col. 1, we provide the catalogue source number. Columns 2-4 provide the 2MASS-corrected OM right ascension and declination and the positional error $\Delta$ (including the rms error on the boresight shift, which was added in quadrature to the statistical 1-sigma positional error calculated for each source). Column 5 provides the XEST OM identification in the $\mathrm{xx}-\mathrm{OM}-\mathrm{zzz}$ notation in Col. 2, where $\mathrm{xx}$ refers to the XEST exposure and zzz refers to the OM identification for this XEST exposure. Columns 6 and 7 give the 2MASS crossidentification, if found, and the offset $\rho_{1}$ between the OM corrected coordinates and the 2MASS coordinates. Columns 8 and 9 give the XEST X-ray identification (in xx-yyy notation, see Güdel et al. 2007a), if found, and the offset $\rho_{2}$ between the OM and X-ray corrected coordinates. Column 10 gives the average magnitude and its uncertainty, and Col. 11 the detection significance. For clarity, we have added in the exponent a $\neq$ character if the filter was $U V W 2$ and a $\dagger$ character if the filter was $U V W 1$. No special character was added for the commonly used $U$ filter. We provide the full OM catalogue as online material at the CDS (Table A.1).

The XEST OM catalogue contains 2148 entries, among which 1893 have 2MASS counterparts and 98 have XEST X-ray counterparts. Out of these 98 sources, 51 are TMC members according to the master list from Güdel et al. (2007a) and 12 are new TMC candidates identified by Scelsi et al. (2007). Thus 35 OM sources had X-ray counterparts but were not classified as TMC members or candidates. Note that V410 Tau ABC (XEST23-032 \& XEST-24-028) was counted twice in the OM catalogue since it was observed in two different XEST observations. In addition, UZ Tau E+W(AB) (XEST-19-049) were not separated in X-rays but were so in the OM (UZ Tau W = XEST-19-OM-092, UZ Tau E = XEST-19-OM-094). Similarly, the X-ray source XEST-08-051 was attributed to both HP Tau/G3 AB and HP Tau/G2 (centroid fitting suggests that the X-rays come mostly from HP Tau/G2); however, they were separated in the OM data (XEST-08-OM-038 for HP Tau/G3 AB and XEST-08-OM-040 for HP Tau/G2).

There were 2 TMC members detected in the OM but not detected in X-rays with XMM-Newton (FV Tau/c AB, XEST02-OM-020 and 2MASS J04141188+2811535, XEST-20-OM002). However, FV Tau/c AB is very close to FV Tau AB (XEST02-013 = XEST-02-OM-017) and its faint X-ray emission may have been overshadowed by the stronger X-ray flux of FV Tau AB. Note that FV Tau/c AB was detected by Chandra (Güdel et al. 2007a). A similar case occurred for the brown dwarf 2MASS J04141188+2811535 (Luhman 2004), which is close to the X-ray bright V773 Tau (XEST-20-042 = XEST-20-OM003). The OM data of the brown dwarf is addressed in a separate paper (Grosso et al. 2007a).

While the above detection numbers start from OM detections, we have also taken a different approach, starting from the 
Table 3. XEST OM catalogue (first thirty entries).

\begin{tabular}{|c|c|c|c|c|c|c|c|c|c|c|}
\hline \# & $\begin{array}{l}\mathrm{RA}_{\text {corr }} \\
\mathrm{h} \mathrm{m} \mathrm{s}\end{array}$ & $\begin{array}{l}\delta_{\text {corr }}, 1,1 \\
0,11\end{array}$ & $\begin{array}{c}\Delta \\
\left({ }^{\prime \prime}\right)\end{array}$ & $\begin{array}{c}\text { XEST } \\
\text { OM }\end{array}$ & 2MASS & $\begin{array}{l}\rho_{1} \\
\left({ }^{\prime \prime}\right)\end{array}$ & XEST & $\begin{array}{l}\rho_{2} \\
\left({ }^{\prime \prime}\right)\end{array}$ & $\overline{\text { Mag }^{a}}$ & Signif. \\
\hline 1 & 040359.95 & +262331.8 & 0.60 & 06-OM-001 & $04035989+2623319$ & 0.73 & & & $20.62 \pm 0.09$ & 15.2 \\
\hline 2 & 040400.62 & +262443.9 & 0.60 & 06-OM-002 & $04040056+2624441$ & 0.81 & & & $19.05 \pm 0.04$ & 21.4 \\
\hline 3 & 040400.78 & +262329.8 & 0.57 & 06-OM-003 & $04040075+2623295$ & 0.45 & & & $16.79 \pm 0.01$ & 114.3 \\
\hline 4 & 040402.24 & +262459.7 & 0.57 & 06-OM-004 & $04040217+2624595$ & 0.84 & & & $16.02 \pm 0.00$ & 176.6 \\
\hline 5 & 040403.27 & +262224.6 & 0.63 & 06-OM-005 & $04040327+2622244$ & 0.13 & & & $19.95 \pm 0.09$ & 10.4 \\
\hline 6 & 040403.59 & +262502.8 & 0.60 & 06-OM-006 & & & & & $18.33 \pm 0.03$ & 38.5 \\
\hline 7 & 040404.69 & +262448.7 & 0.63 & 06-OM-007 & $04040467+2624478$ & 0.84 & & & $20.15 \pm 0.10$ & 8.5 \\
\hline 8 & 040407.46 & +261910.4 & 0.61 & 06-OM-008 & $04040741+2619102$ & 0.60 & & & $19.19 \pm 0.04$ & 19.6 \\
\hline 9 & 040408.17 & +261750.7 & 0.59 & 06-OM-009 & $04040814+2617508$ & 0.33 & & & $18.38 \pm 0.01$ & 37.5 \\
\hline 10 & 040408.31 & +261918.6 & 0.63 & 06-OM-010 & & & & & $20.46 \pm 0.17$ & 6.2 \\
\hline 11 & 040408.43 & +261949.0 & 0.63 & 06-OM-011 & & & & & $20.15 \pm 0.12$ & 8.4 \\
\hline 12 & 040409.26 & +261910.1 & 0.61 & 06-OM-012 & $04040919+2619098$ & 0.88 & & & $19.26 \pm 0.12$ & 18.1 \\
\hline 13 & 040409.35 & +261406.2 & 0.59 & 06-OM-013 & $04040936+2614059$ & 0.32 & & & $18.65 \pm 0.03$ & 29.7 \\
\hline 14 & 040409.50 & +261516.7 & 0.64 & 06-OM-014 & & & & & $20.87 \pm 0.08$ & 4.4 \\
\hline 15 & 040409.57 & +262203.8 & 0.58 & 06-OM-015 & $04040952+2622034$ & 0.62 & & & $17.38 \pm 0.02$ & 78.6 \\
\hline 16 & 040409.87 & +261900.6 & 0.64 & 06-OM-016 & & & & & $20.51 \pm 0.16$ & 6.0 \\
\hline 17 & 040410.24 & +262158.7 & 0.60 & 06-OM-017 & $04041018+2621581$ & 0.98 & & & $18.94 \pm 0.05$ & 24.4 \\
\hline 18 & 040410.44 & +262257.3 & 0.64 & 06-OM-018 & & & & & $20.84 \pm 0.14$ & 4.7 \\
\hline 19 & 040410.53 & +261901.3 & 0.58 & 06-OM-019 & $04041051+2619011$ & 0.33 & & & $17.67 \pm 0.03$ & 63.8 \\
\hline 20 & 040411.02 & +261057.7 & 0.61 & 06-OM-020 & $04041104+2610573$ & 0.51 & & & $19.19 \pm 0.06$ & 19.6 \\
\hline 21 & 040411.19 & +262004.0 & 0.61 & 06-OM-021 & $04041116+2620036$ & 0.50 & & & $19.57 \pm 0.05$ & 14.3 \\
\hline 22 & 040411.22 & +261047.7 & 0.63 & 06-OM-022 & & & & & $20.21 \pm 0.19$ & 7.7 \\
\hline 23 & 040411.34 & +262516.4 & 0.57 & 06-OM-023 & $04041129+2625162$ & 0.65 & & & $16.77 \pm 0.01$ & 115.6 \\
\hline 24 & 040411.82 & +261141.1 & 0.64 & 06-OM-024 & & & & & $20.92 \pm 0.17$ & 4.1 \\
\hline 25 & 040411.83 & +262503.8 & 0.62 & 06-OM-025 & $04041178+2625035$ & 0.64 & & & $19.62 \pm 0.13$ & 13.3 \\
\hline 26 & 040411.85 & +261203.2 & 0.62 & 06-OM-026 & & & & & $19.63 \pm 0.09$ & 13.5 \\
\hline 27 & 040411.89 & +261124.4 & 0.59 & 06-OM-027 & $04041191+2611239$ & 0.58 & & & $18.59 \pm 0.04$ & 32.1 \\
\hline 28 & 040411.93 & +261912.0 & 0.60 & 06-OM-028 & $04041190+2619118$ & 0.25 & & & $19.00 \pm 0.05$ & 22.8 \\
\hline 29 & 040412.66 & +261244.4 & 0.63 & 06-OM-029 & & & $06-029$ & 2.86 & $20.13 \pm 0.12$ & 8.7 \\
\hline 30 & 040413.03 & +261141.6 & 0.58 & 06-OM-030 & $04041305+2611414$ & 0.27 & & & $18.00 \pm 0.02$ & 50.3 \\
\hline
\end{tabular}

${ }^{a} U$-band except if identified with ${ }^{\dagger}(U V W 1)$ or ${ }^{\ddagger}(U V W 2)$.

Table 4. Detection statistics.

\begin{tabular}{lrrrrrrrr}
\hline \hline & $\#$ & \multicolumn{7}{c}{ Type $^{a}$} \\
& & 0 & 1 & 2 & 3 & 4 & 5 & 9 \\
\hline Detected OM sources & 2148 & & & & & & \\
X-ray sources in OM FOV & 916 & & & & & & & \\
OM detections not detected in X-rays & 2 & 0 & 0 & 1 & 0 & 1 & 0 & 0 \\
OM detections also detected in X-rays & 98 & & & & & & & \\
TMC members detected in X-rays and OM & 51 & 0 & 1 & 33 & 17 & 0 & 0 & 0 \\
TMC candidates detected in X-rays and OM & 12 & & & & & & & \\
TMC members detected in X-rays but not in OM & 34 & 0 & 7 & 8 & 8 & 4 & 0 & 7 \\
TMC candidates detected in X-rays but not in OM & 21 & & & & & & \\
TMC members detected in X-rays but outside $\mathrm{OM} \mathrm{FOV}^{b}$ & 55 & 0 & 1 & 16 & 32 & 4 & 2 & 0 \\
\hline
\end{tabular}

${ }^{a}$ See Güdel et al. (2007a) and text for the definition of the object type. ${ }^{b}$ Statistics for XEST observations with OM data.

catalog of X-ray sources detected with XEST: we have determined that $916 \mathrm{X}$-ray sources had coordinates such that they fell on the OM detector. Out of those, only 98 are detected in the OM (see above), while 34 TMC members of TMC detected in X-rays remained undetected in the OM. In addition, 21 new TMC candidates detected in X-rays (Scelsi et al. 2007) were not detected in the OM. Table 4 summarizes the detection statistics pertaining to the OM/X-ray correlations. The object type (as defined by Güdel et al. 2007a, i.e., "0" and " 1 " for a protostar of Class 0 or Class I, "2" for an accreting (classical) T Tau star usually showing a Class-II IR spectrum, "3" for a weak-lined or Class-III object, "4" for a brown dwarf, "5" for an Herbig Ae star, and "9" for uncertain classifications or other object types) is given when relevant.
No Type 0 object was in our sample since the sole object surveyed was observed by Chandra only and remained undetected in X-rays (L1527 IRS; Güdel et al. 2007a). For TMC members detected in the X-rays and falling on the OM FOVs, the OM detection rate was $12.5 \%$ ( 1 out of 8 ) for Type 1 objects, whereas this rate reached $80.5 \%$ in type 2 objects. Type 3 objects had a high $68 \%$ detection rate. Interestingly, only 1 brown dwarf was detected in the OM, whereas 11 such objects fell on the OM FOV and 4 of them were detected in X-rays (Grosso et al. 2007a,b). We remind that the numbers reported in Table 4 makes no attempt not to count duplicates. For example, CFHTTau 5, detected in X-rays in two XEST observations (XEST-03031 = XEST-04-003), was counted once in "TMC X-ray OM non-detections" since it fell on the OM detector but remained 
Table 5. XEST OM catalogue of TMC members detected in X-rays and in the OM.

\begin{tabular}{|c|c|c|c|c|c|c|c|c|c|}
\hline$\overline{\mathrm{RA}_{\text {corr }}}$ & $\delta_{\text {corr }}$ & XEST OM & XEST & Name & Type & $\operatorname{Mag}^{a}$ & Rate $_{\mathrm{OM}}$ & $\overline{L_{\mathrm{X}}^{b}}$ & Rate $_{X}$ \\
\hline 041412.92 & +281212.6 & $20-\mathrm{OM}-003$ & $20-042$ & V773 Tau ABC & 3 & $13.16 \pm 0.02$ & 109.13 & $\frac{x}{9.49}$ & 1.2113 \\
\hline 041413.58 & +281249.4 & 20-OM-004 & $20-043$ & FM Tau & 2 & $14.44 \pm 0.07$ & 33.76 & 0.53 & 0.0611 \\
\hline 041417.02 & +281057.9 & 20-OM-005 & $20-046$ & CW Tau & 2 & $15.50 \pm 0.10$ & 12.73 & 2.84 & 0.0015 \\
\hline 041417.66 & +280609.8 & 20-OM-006 & $20-047$ & CIDA 1 & 2 & $18.04 \pm 0.15$ & 1.23 & 0.03 & 0.0031 \\
\hline 041831.10 & +282716.2 & 23-OM-001 & $23-032$ & V410 Tau ABC & 3 & $15.85 \pm 0.15^{\ddagger}$ & 0.38 & 3.76 & 1.2910 \\
\hline 041831.10 & +282716.2 & 24-OM-001 & $24-028$ & V410 Tau ABC & 3 & $15.69 \pm 0.11^{\ddagger}$ & 0.44 & 4.66 & 0.8534 \\
\hline 041915.84 & +290626.7 & 28-OM-014 & $28-100$ & BP Tau & 2 & $13.01 \pm 0.10^{\dagger}$ & 46.80 & 1.37 & 0.2343 \\
\hline 042143.27 & +193412.9 & $01-\mathrm{OM}-022$ & $01-028$ & IRAS 04187+1927 & 2 & $19.91 \pm 0.31^{\dagger}$ & 0.08 & 0.91 & 0.0481 \\
\hline 042159.45 & +193206.4 & 01-OM-039 & $01-045$ & T Tau N(+Sab) & 2 & $12.00 \pm 0.04^{\dagger}$ & 119.13 & 8.05 & 0.8189 \\
\hline 042202.17 & +265731.5 & 11-OM-087 & $11-057$ & FS Tau AC & 2 & $17.57 \pm 0.00$ & 1.88 & 3.22 & 0.1449 \\
\hline 042204.86 & +193448.3 & 01-OM-042 & 01-054 & RX J0422.1+1C & 3 & $19.58 \pm 0.08^{\dagger}$ & 0.11 & 3.11 & 0.3390 \\
\hline 042216.76 & +265456.6 & $11-\mathrm{OM}-126$ & $11-079$ & CFHT-Tau 21 & 2 & $18.78 \pm 0.18$ & 0.62 & 0.15 & 0.0070 \\
\hline 042653.56 & +260654.6 & 02-OM-017 & $02-013$ & FV Tau AB & 2 & $19.58 \pm 0.09$ & 0.30 & 0.53 & 0.0212 \\
\hline 042704.69 & +260615.9 & $02-\mathrm{OM}-028$ & $02-022$ & DG Tau A & 2 & $13.57 \pm 0.05$ & 75.08 & $0.47^{c}$ & 0.0290 \\
\hline 042920.73 & +263340.3 & $15-\mathrm{OM}-035$ & $15-020$ & JH 507 & 3 & $17.35 \pm 0.04$ & 2.30 & 0.46 & 0.0382 \\
\hline 042923.71 & +243300.2 & 13-OM-001 & $13-004$ & GV Tau AB & 1 & $19.20 \pm 0.06$ & 0.42 & $0.95^{c}$ & 0.0293 \\
\hline 042941.59 & +263258.0 & $15-\mathrm{OM}-112$ & $15-040$ & DH Tau AB & 2 & $14.79 \pm 0.04$ & 24.38 & 8.46 & 1.0729 \\
\hline 042942.52 & +263249.1 & $15-\mathrm{OM}-118$ & $15-042$ & DI Tau AB & 3 & $15.99 \pm 0.01$ & 8.11 & 1.57 & 0.0979 \\
\hline 043044.25 & +260124.4 & $14-\mathrm{OM}-258$ & $14-057$ & DK Tau AB & 2 & $14.09 \pm 0.14$ & 46.54 & 0.92 & 0.0989 \\
\hline 043140.07 & +181357.2 & 22-OM-002 & $2-047$ & XZ Tau AB & 2 & $16.70 \pm 0.15^{\ddagger}$ & .17 & 0.96 & 0.2440 \\
\hline 043150.61 & +242417.7 & 3-OM-001 & $3-005$ & HK Tau AB & 2 & $16.81 \pm 0.09$ & .81 & 0.08 & 0.0045 \\
\hline 043215.39 & +242859.3 & 3-OM-007 & 03-016 & Haro 6 & 2 & 19. & .41 & 0.80 & 0.0141 \\
\hline 043218.84 & +242 & 3-OM-008 & 03-019 & V928 & 3 & 17. & .76 & .05 & 0732 \\
\hline 043230.58 & +241957.4 & 03-OM-015 & -022 & FY Tau & 2 & $17.83 \pm$ & 48 & 81 & 0.0822 \\
\hline 043231.77 & +2420( & -016 & -023 & FZ Tau & 2 & $16.15 \pm$ & 6.99 & 0.64 & 0.0232 \\
\hline 043242.84 & +255231.3 & 19-OM-092 & $19-049$ & UZ Tau W & 2 & $14.23 \pm$ & 40.86 & 0.89 & 0.0465 \\
\hline 043243.05 & +255231.1 & 19-OM-094 & $19-049$ & UZ Tau E & 2 & $14.33 \pm 0$ & 37.37 & 0.89 & 0.0465 \\
\hline 043249.10 & +225303.0 & 17-OM-001 & $17-009$ & JH 112 & 2 & $18.81 \pm 0$ & 0.60 & 0.82 & 0.0357 \\
\hline 043334.05 & +242116.9 & 04-OM-018 & 04-034 & GI Tau & 2 & $14.34 \pm 0.07$ & 36.97 & 0.83 & 0.0364 \\
\hline 043334.54 & +242105.6 & 04-OM-020 & $04-035$ & GK Tau AB & 2 & $15.50 \pm 0.09$ & 12.73 & 1.47 & 0.1404 \\
\hline 043336.80 & +260949.6 & 18-OM-002 & $18-019$ & IS Tau AB & 0 & $18.52 \pm 0.11$ & 0.79 & 0.66 & 0.0586 \\
\hline 043351.98 & +225030.3 & 17-OM-066 & $17-058$ & CI Tau & 0 & $14.06 \pm 0.08$ & 48.00 & 0.19 & 0.0204 \\
\hline 043354.61 & +261326.8 & 18-OM-004 & $18-030$ & IT Tau AB & 2 & $18.01 \pm 0.05$ & 1.25 & 6.49 & 0.4041 \\
\hline 043455.42 & +242852.7 & 25-OM-003 & 5-026 & AA Tau & 2 & $15.67 \pm 0.18^{\ddagger}$ & 0.45 & 1.24 & 0.0597 \\
\hline 043520.93 & +225423.4 & 08-OM-005 & 08-019 & FF Tau AB & ? & $17.57 \pm 0.03$ & 1.89 & 0.80 & 0.0681 \\
\hline 043527.39 & +241458.5 & 12-OM-077 & $12-040$ & DN Tau & ? & $13.27 \pm 0.04$ & 98.91 & 1.15 & 0.1955 \\
\hline 043540.94 & +241109.2 & $12-\mathrm{O}$ & $12-059$ & $\mathrm{CoKu} \mathrm{Ta}$ & 3 & \pm 0.60 & 0.12 & 5.85 & 0.5224 \\
\hline 043541.80 & +223411.8 & $09-\mathrm{O}$ & 09-022 & Tau 8 & 3 & $4 \pm 0.04$ & 0.11 & 0.50 & 0.0668 \\
\hline 043547.36 & +225020.6 & 032 & 08-037 & & 2 & $1 \pm 0.04$ & 20.03 & 2.48 & 0.3050 \\
\hline 043551.10 & +22523 & 34 & & & 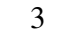 & 19 & 0.25 & 2.62 & 0.2232 \\
\hline 043 & +225422.7 & & & & 2 & 17.3 & 2.22 & 2.55 & 0.1156 \\
\hline 043553.52 & +225408.6 & $08-\mathrm{O}$ & $08-$ & HP Ta & 3 & $18.21 \pm$ & 1.05 & 1.29 & 0.0648 \\
\hline 043554.16 & +225412.9 & 08-OM-040 & $08-051$ & HP Tau/G2 & 5 & $13.63 \pm$ & 70.80 & 9.65 & 0.8136 \\
\hline 043556.84 & +225434.9 & 08-OM-044 & $08-058$ & Haro 6-28 AB & 2 & $18.94 \pm$ & 0.53 & 0.25 & 0.0124 \\
\hline 043920.87 & +254502.8 & 05-OM-002 & $05-013$ & GN Tau AB & 2 & $17.22 \pm 0.11$ & 2.61 & 0.79 & 0.0108 \\
\hline 044049.61 & +255119.2 & 07-OM-006 & 07-011 & JH 223 & 3 & $18.25 \pm 0.04$ & 1.01 & 0.06 & 0.0131 \\
\hline 044205.46 & +252256.0 & 10-OM-002 & $10-017$ & CoKuLk332/G2 & 3 & $18.68 \pm 0.06$ & 0.68 & 3.26 & 0.1826 \\
\hline 044207.33 & +252303.8 & $10-\mathrm{OM}-003$ & $10-018$ & CoKuLk332/G1 A & 3 & $19.09 \pm 0.15$ & 0.47 & 0.49 & 0.0470 \\
\hline 044207.77 & +252312.3 & $10-\mathrm{OM}-004$ & $10-020$ & V955 Tau AB & 2 & $17.90 \pm 0.08$ & 1.39 & 1.62 & 0.0576 \\
\hline 044221.06 & +252034.1 & 10-OM-006 & $10-034$ & CIDA 7 & 2 & $18.67 \pm 0.09$ & 0.68 & 0.04 & 0.0028 \\
\hline 044237.66 & +251537.2 & $10-\mathrm{OM}-010$ & $10-045$ & DP Tau & 2 & $15.84 \pm 0.03$ & 9.29 & $0.10^{c}$ & 0.0031 \\
\hline
\end{tabular}

${ }^{a} U$-band except if identified with ${ }^{\dagger}(U V W 1)$ or ${ }^{\ddagger}(U V W 2) .{ }^{b} L_{\mathrm{X}}(0.3-10 \mathrm{keV})$ in $10^{30} \mathrm{erg} \mathrm{s}^{-1}$ from the DEM method. ${ }^{c} L_{\mathrm{X}}(0.3-10 \mathrm{keV})$ of the hard component from Güdel et al. (2007b).

undetected in XEST-03, and once in "TMC X-ray outside OM FOV" since it fell off the OM detector in XEST-04.

Tables 5 and 6 repeat some of the columns in Table A.1 for TMC members and TMC candidates, respectively, but also provide the name of the TMC source (from Güdel et al. 2007a) or "(TMC cand)" (from Scelsi et al. 2007). The average OM count rate Rate OM $_{\text {(corrected for coincidence losses and aperture }}$ radius) is also given after calculation from the magnitude ${ }^{2}$. We used the zero-point magnitudes given in the XMM-Newton UHB $\left(Z_{U}=18.2593, Z_{U V W 1}=17.1882, Z_{U V W 2}=14.8026\right.$; Ehle et al. 2005). Note that this calibration does not take into account corrections specific to the source's spectral type. We also provide the X-ray luminosity $L_{X}$ and X-ray count rate $\left(\right.$ Rate $\left._{X}\right)$ from Güdel et al. (2007a).

\footnotetext{
${ }^{2} \log$ Rate $_{\mathrm{OM}}=-0.4(m-Z)$, where $m$ is the source's magnitude, and $Z$ is the zero-point magnitude.
} 
Table 6. XEST OM catalogue of TMC candidates detected in X-rays and in the OM.

\begin{tabular}{rcccccrrr}
\hline \hline RA $_{\text {corr }}$ & $\delta_{\text {corr }}$ & XEST OM & XEST & Name & Mag $^{a}$ & Rate $_{\text {OM }}$ & $L_{\mathrm{X}}^{b}$ & Rate $_{\mathrm{X}}$ \\
\hline 040424.45 & +261112.0 & 06-OM-079 & $06-041$ & (TMC cand) & $18.02 \pm 0.05$ & 1.25 & 7.3 & 17.1 \\
041452.31 & +280559.7 & 20-OM-010 & $20-071$ & (TMC cand) & $19.87 \pm 0.25$ & 0.23 & 171 & 159 \\
042215.72 & +265705.3 & $11-O M-122$ & $11-078$ & (TMC cand) & $20.98 \pm 0.14$ & 0.08 & 23 & 9.4 \\
042227.29 & +265949.4 & $11-O M-150$ & $11-088$ & (TMC cand) & $20.11 \pm 0.08$ & 0.18 & 1.6 & 2.1 \\
042935.98 & +243557.0 & 13-OM-002 & $13-010$ & (TMC cand) & $20.47 \pm 0.23$ & 0.13 & 68 & 22.1 \\
042936.29 & +263423.2 & $15-O M-093$ & $15-034$ & (TMC cand) & $18.70 \pm 0.11$ & 0.66 & 10 & 24.4 \\
043025.21 & +260256.9 & $14-O M-163$ & $14-034$ & (TMC cand) & $16.86 \pm 0.01$ & 3.62 & 0.35 & 0.8 \\
043333.07 & +225250.7 & 17-OM-028 & $17-043$ & (TMC cand) & $20.66 \pm 0.36$ & 0.11 & 1.2 & 20.4 \\
043352.54 & +225627.0 & 17-OM-070 & $17-059$ & (TMC cand) & $19.12 \pm 0.06$ & 0.45 & 40 & 57.4 \\
043355.64 & +242501.7 & 04-OM-044 & $04-060$ & (TMC cand) & $17.30 \pm 0.01$ & 2.42 & 0.74 & 1.3 \\
043552.88 & +225057.8 & 08-OM-037 & $08-049$ & (TMC cand) & $20.06 \pm 0.21$ & 0.19 & 115 & 118 \\
043558.93 & +223835.2 & 09-OM-143 & $09-042$ & (TMC cand) & $15.37 \pm 0.03$ & 14.29 & 172 & 269 \\
\hline
\end{tabular}

${ }^{a} U$-band. ${ }^{b} L_{\mathrm{X}}(0.3-10 \mathrm{keV})$ in $10^{30} \mathrm{erg} \mathrm{s}^{-1}$ from the DEM method.

\section{Properties of the OM Taurus sample}

We focus in this section on some of the OM properties of the TMC sample as defined in Güdel et al. (2007a) that were detected in both X-rays and in the OM. Specifically, we focus on whether correlations can be found, e.g., between the X-ray luminosity and the OM magnitude, and on the $U$-band properties of weak-lined $\mathrm{T}$ Tau stars and classical T Tau stars. The low number of detections of type 1 sources is such that we will not discuss them.

Figure 2 shows the scatter plot of X-ray luminosities (as derived from the DEM method; see Güdel et al. 2007a) as a function of the observed OM magnitude ( $U$-band or ultraviolet $U V W 1$ and $U V W 2$ bands). We used different symbols for the various types of stars. There is no strong evidence that any correlation exists in any type or any $\mathrm{OM}$ filter. However, at least one trend can be noticed: type 2 stars (usually CTTS) are overall fainter in X-rays than type 3 stars (usually WTTS). At a given OM magnitude, there is a wide range of X-ray luminosities below $10^{31} \mathrm{erg} \mathrm{s}^{-1}$ in type 2 stars. Figure 3 shows the cumulative distribution of X-ray count rates in type 2 and 3 stars that were detected in both OM and X-rays. A Kolmogorov-Smirnov (K-S) test gives a significance level of $P=0.0055$ that both distributions are compatible ( $D=0.49$; the $D$ statistic is the maximum vertical deviation between the two curves), indicating that the two cumulative distributions differ at a high significance level. This result is discussed in detail in Güdel et al. (2007a) and Telleschi et al. (2007a).

Despite the clear difference in X-ray luminosity between type 2 and 3 stars, there is little evidence that the observed OM magnitude of type 2 stars are much different from those of type 3 stars (Fig. 4). Indeed, a K-S test gives a significance level of $P=0.54(D=0.25)$. If the observed OM magnitudes scale with $L_{*}$, then the similar distributions for type 2 and 3 stars would not be surprising, and again, we would find the CTTS to be fainter only in the X-rays (Fig. 3), pointing at a true deficiency of emission in that wavelength range (Telleschi et al. 2007a). However, it is important to keep in mind that the observed magnitudes could be biased by certain factors. Therefore, we aim to test the following hypotheses that could produce an observational bias: I) Bolometric luminosities in type 2 and type 3 stars are different; II) The binary fraction is higher in one object type than another; III) Extinction in the $U$ band is stronger for one type than for the other; IV) One object type has a significant excess in the $U$-band.

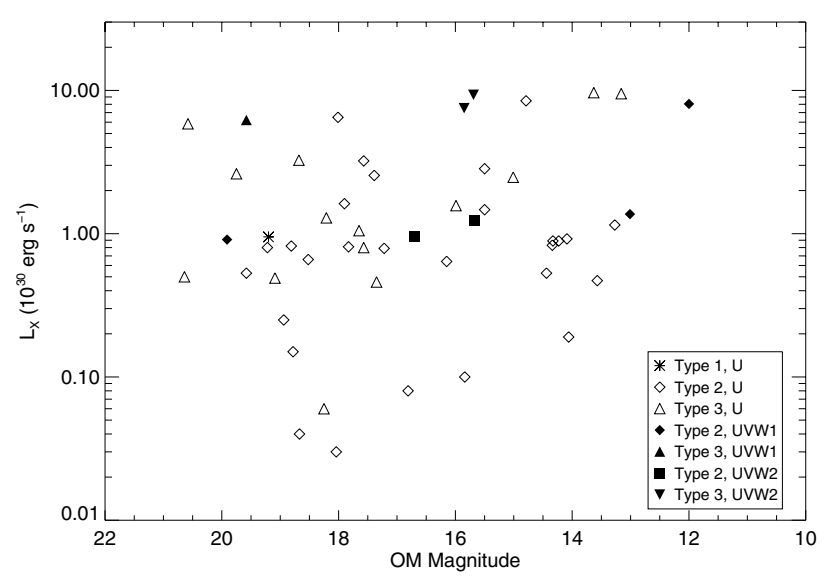

Fig. 2. X-ray luminosity $\left(L_{\mathrm{X}}\right)$ in $10^{30} \mathrm{erg} \mathrm{s}^{-1}$ as a function of the observed OM magnitude. Various types of stars are identified (see text). Ultraviolet magnitudes are identified with filled symbols.

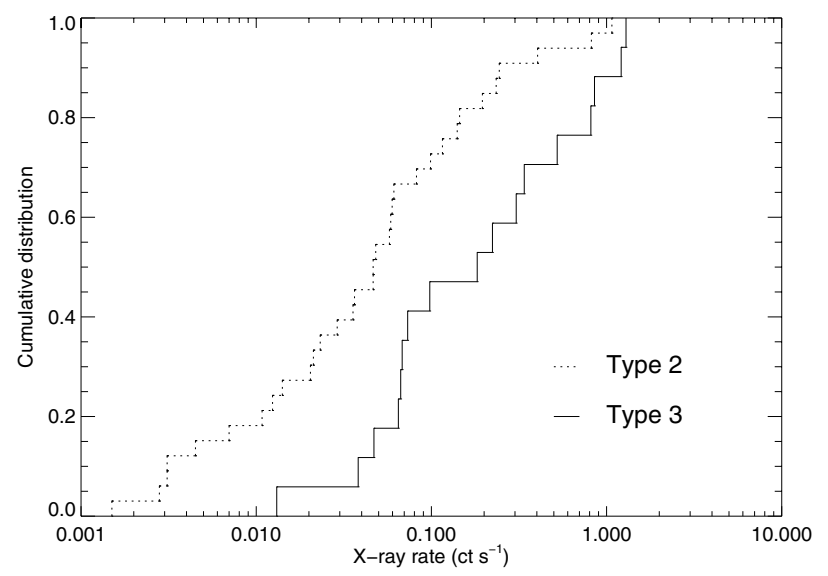

Fig. 3. Cumulative distributions of X-ray count rates of type 2 and 3 stars.

\subsection{Hypothesis I}

Figure 5 shows the cumulative distribution of the star's luminosity (see Güdel et al. 2007a) for type 2 and 3 stars detected in the $U$ band. Note that we used the luminosity of the primary component if available for multiples (Table 9 in Güdel et al. 2007a). There is no evidence that the stellar luminosity 


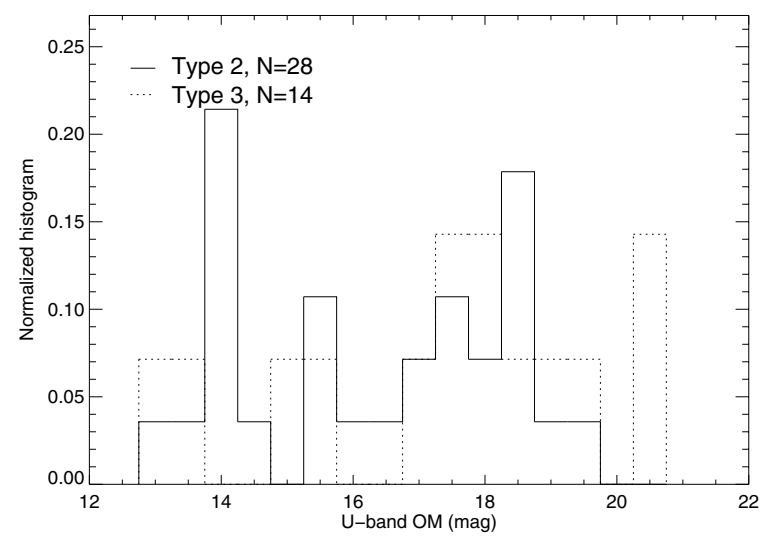

Fig. 4. Histogram of OM $U$ magnitudes for type 2 and 3 stars.

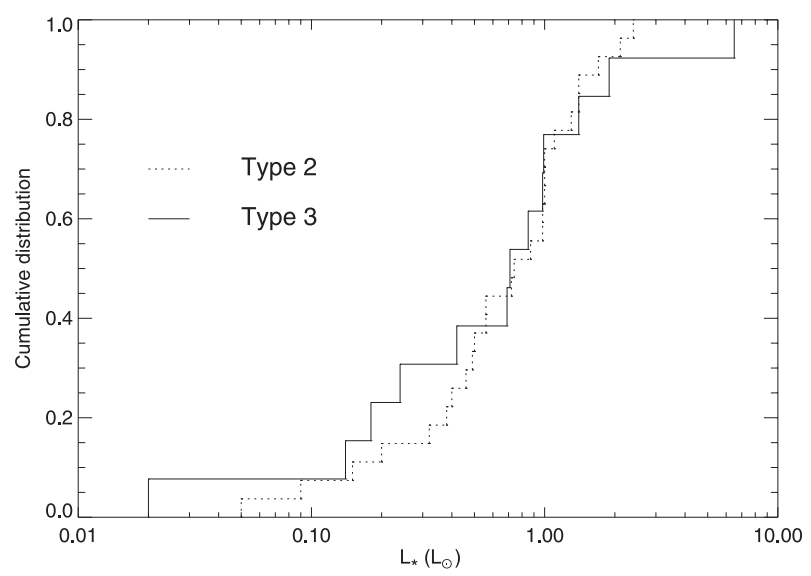

Fig. 5. Cumulative distributions of stellar luminosity of type 2 and 3 objects detected in the $U$ filter.

of type 2 stars is different from that of type 3 stars, as indicated by the high significance of a K-S test $(D=0.16, P=0.97)$. Therefore, hypothesis I can be safely rejected as a potential biasing factor.

\subsection{Hypothesis II}

In our OM $U$-band sample, there are 14 and 9 TMC stars of type 2 and 3 , respectively, with more than 1 component. Therefore, we created cumulative distributions of the OM magnitude for the remaining single components (14 and 5 stars for type 2 and 3, respectively): There is no evidence of a difference in the OM magnitudes for single type 2 and 3 stars (K-S test: $D=0.37, P=0.58$ ). Therefore, hypothesis II is also rejected.

\subsection{Hypothesis III}

We used extinction magnitudes in the $J$ band $\left(A_{J}\right)$ if available, and determined the $A_{U}$ magnitudes. Our procedure was the following: first, we calculated $A_{J} / A_{V}$ and $A_{U} / A_{V}$ ratios based on coefficients given by Cardelli et al. (1989): $A_{J} / A_{V}=0.4008-$ $0.3679 / R_{V}$ and $A_{U} / A_{V}=0.9530+1.9090 / R_{V}$, where we used $R_{V}=5.5$. Indeed such a value gives a better fit of the observed

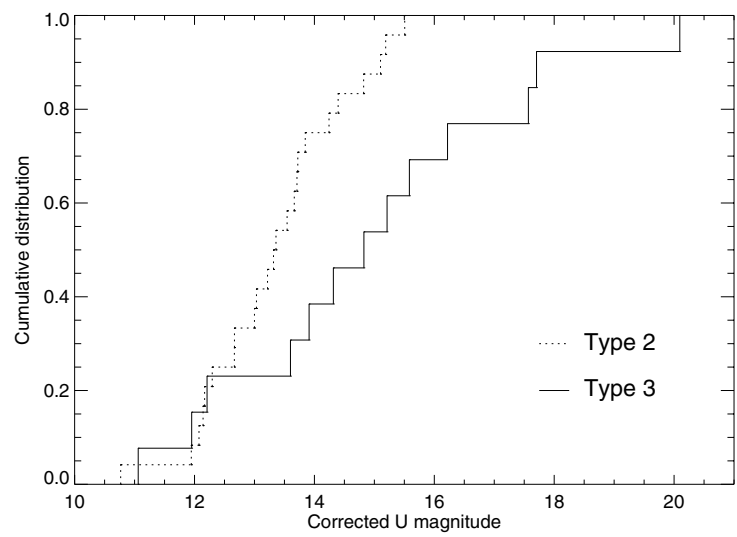

Fig. 6. Cumulative distributions of $U$-band OM magnitudes after correction for extinction. Type 2 stars have brighter OM magnitudes than type 3 stars. Stars with known $T_{\text {eff }}$ and $A_{J}$ are shown.

$A_{J} / A_{V}$ values $^{3}$. Then, we calculate the estimated extinction magnitude in the $U$ band, $A_{U}$ :

$A_{U}=\frac{A_{U}}{A_{V}} \frac{A_{V}}{A_{J}} A_{J}$

We obtained the distributions of $A_{U}$ for type 2 and 3 stars observed with the $\mathrm{OM}$ in the $U$ band. Unexpectedly, the cumulative distributions do not differ significantly. To verify this finding, we calculated $A_{U}$ from $A_{V}\left(A_{U}=\frac{A_{U}}{A_{V}} A_{V}\right)$ and found the same result. Since $A_{V}$ is more subject to optical veiling than $A_{J}$, we preferred to use $A_{U}$ obtained from $A_{J}$ in this paper.

Although the distributions of extinction magnitudes are overall similar in our sample, we have noticed that they are correlated with the observed $U$-band magnitude in type 2 stars, but not in type 3 stars. It appears that, in our sample, type 2 stars with fainter $U$ magnitudes suffer from more extinction than brighter type 2 stars. Therefore, we have used the above $A_{U}$ to correct the observed OM $U$-band magnitudes and we constructed the cumulative distribution (Fig. 6). The distributions are significantly different, with type 2 stars being much brighter in the $U$-band than type 3 stars (K-S statistic $D=0.44$ with a significance level $P$ of $0.05)$.

Therefore, hypothesis III appears to be correct in the sense that type 2 stars are brighter in the $U$-band but suffer more extinction than type 3 stars, as could be expected based on observed properties of young stars. We emphasize, however, that this trend is opposite to the trend in X-rays where CTTS are fainter. Nevertheless, as shown earlier, both types of stars have similar stellar luminosities, indicating that there must be a $U$-band excess in type 2 stars. Therefore, we need to address hypothesis IV before we can draw any firm conclusion.

\subsection{Hypothesis IV}

To determine the $U$-band excesses, we used the distance modulus ( $m-M=5.73$ for a distance to the TMC of $140 \mathrm{pc}$ ) to calculate the corrected absolute $U$-band magnitude, $M_{U}$, from the observed extinction-corrected $U$ magnitude. We then determined the stellar photospheric absolute $U$ magnitude tabulated by Siess et al. (2000) (that use a $Z=0.02$ metallicity and the

3 When more than one extinction magnitude was available for multiples, we used the value from the primary. 

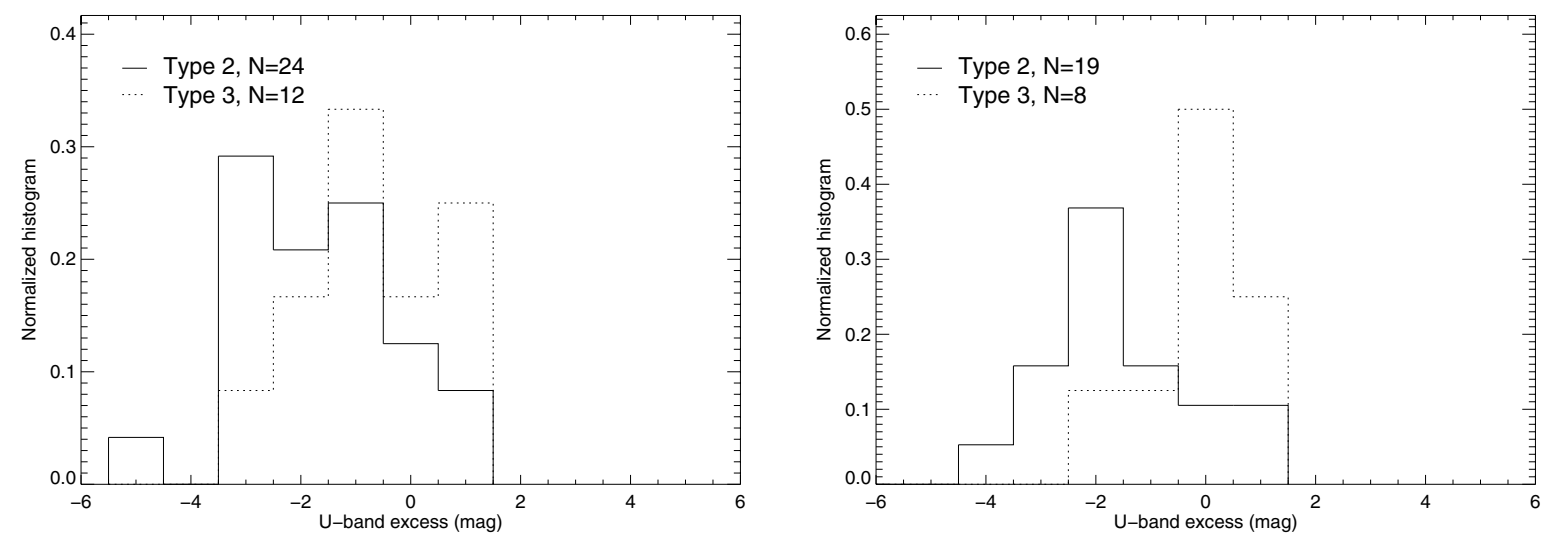

Fig. 7. Normalized distributions of $U$-band excesses for type 2 and 3 stars. Excesses determined when $A_{J}$ was used to calculate $A_{U}$ are shown in the left figure, whereas excesses determined with $A_{V}$ used are shown in the right figure.

conversion table of Kenyon \& Hartmann 1995). We finally compared the stellar photospheric $M_{U}$ with the observed extinctioncorrected absolute $U$ magnitude. Figure 7 shows the normalized histograms of the $U$-band excesses. We provide distributions determined from $A_{U}$ that was calculated either from $A_{J}$ (left figure) or $A_{V}$ (right figure). The distributions of excesses are different, as shown from Kolmogorov-Smirnov tests of the cumulative distributions of excesses that give very low probabilities ( $P=0.16$ and $P=0.04$ for excesses derived from $A_{J}$ and $A_{V}$, respectively). Type 2 stars generally show a $U$-band excess of 0-3 mag, whereas type 3 stars either show no excess or slight $U$-band deficiency. There are exceptions for stars of both types, probably due to inaccurate background subtraction (mostly due to the central ghost emission at the center of the OM image), inaccurate extinction correction, binary effects, or a lower than expected count rate in bright stars due to instrumental effects (fixed pattern noise).

In conclusion, we have shown that binarity and stellar luminosity in our sample do not bias the observed OM magnitudes. However, although the extinction magnitudes are statistically similar in type 2 and 3 stars, they are correlated in type 2 stars with the observed magnitudes. After correction for extinction, type 2 stars are found to be brighter than type 3 stars, which can be explained by a $U$-band excess due to accretion.

\section{Optical and X-ray light curves}

Figure B.1 presents the collection of simultaneous X-ray and optical/UV light curves of XEST sources detected in both X-rays and in the OM. The X-ray light curves are shown on the top panel with the XEST identification, while the OM light curve is shown in the bottom panel with the XEST-OM identification. The OM light curves are taken from the IMAGING mode data only. The $x$-axis OM error bars correspond to the length of an exposure identification. If a TMC member is associated with the source, its name is given next to the XEST or XEST-OM identification. Special cases occur, e.g., when two nearby sources were not separated in X-rays but they were in the OM. For example, the X-ray light curve of XEST-19-049 is associated with both UZ Tau E and W, whereas we manually extracted (due to problems in the SAS software to properly extract both sources due to the close separation) the OM light curves for UZ Tau $\mathrm{W}$ (AB) and UZ Tau E (XEST-19-OM-092 and XEST-19-OM094, respectively $)^{4}$. A similar case is reported for HP Tau/G3 $\mathrm{AB}$ and HP Tau/G2 (XEST-08-051). The X-ray light curve, although dominated by HP Tau/G2, has some contribution (about 8\%; see Telleschi et al. 2007a) from HP Tau/G3 AB. In contrast, the OM cleanly separated their emission (HP Tau/G3 AB = XEST-08-OM-038; HP Tau/G2 = XEST-08-OM-40). TMC candidates (Scelsi et al. 2007) are identified as well. X-ray and OM sources presently not identified as either TMC member or TMC candidate only have their XEST and XEST-OM identifications.

As mentioned earlier, our database includes several cases where only a few OM points were available for a specific target (e.g., CoKu Tau 3 AB; CW Tau; CIDA 1, etc.). However, there are several cases for which we have very good coverage even with the OM IMAGING data (e.g., FM Tau; V773 Tau ABC; CIDA 7; etc.). DG Tau A (XEST-02-022 = XEST-02-OM-028) is a good example where even low time resolution was able to catch the $U$-band emission of a flare, which peaked before the peak in the X-rays, a signature of the Neupert effect. Güdel et al. (2007b) give a full analysis of the DG Tau A case and make use of the OM light curve.

In addition, some OM data were obtained in FAST mode, allowing us to obtain high time resolution light curves. Figure 8 shows the OM FAST light curves together with the X-ray light curves. The classification type is also given on the upper right corner of each panel. The light curve of T Tau will be presented elsewhere (Güdel et al. 2007c). The OM FAST data of IT Tau and UZ Tau EW were not useful since the sources fell on or slightly outside the OM FAST window, and thus the light curves

4 The OM light curves were obtained by manually determining the position of the two stars in each exposure image, and then using an IDL routine to extract the light curve. We used radii of half the stars' separation (3.' 48 , i.e., a radius of 3.65 pixels for the central window), which minimizes the contamination of one source onto the second one. We corrected for aperture, applied the theoretical and empirical corrections, corrected for deadtime, and applied a time-dependent sensitivity correction. The above procedure is similar to what the SAS task omsource does; however, the latter cannot yet extract for radii lower than 6 pixels. We defined an annulus of radii 20 and 30 pixels $\left(\approx 10^{\prime \prime}\right.$ and $\left.15^{\prime \prime}\right)$ for the local background contribution. See Grosso et al. (2007a) for a detailed description of the procedure. 

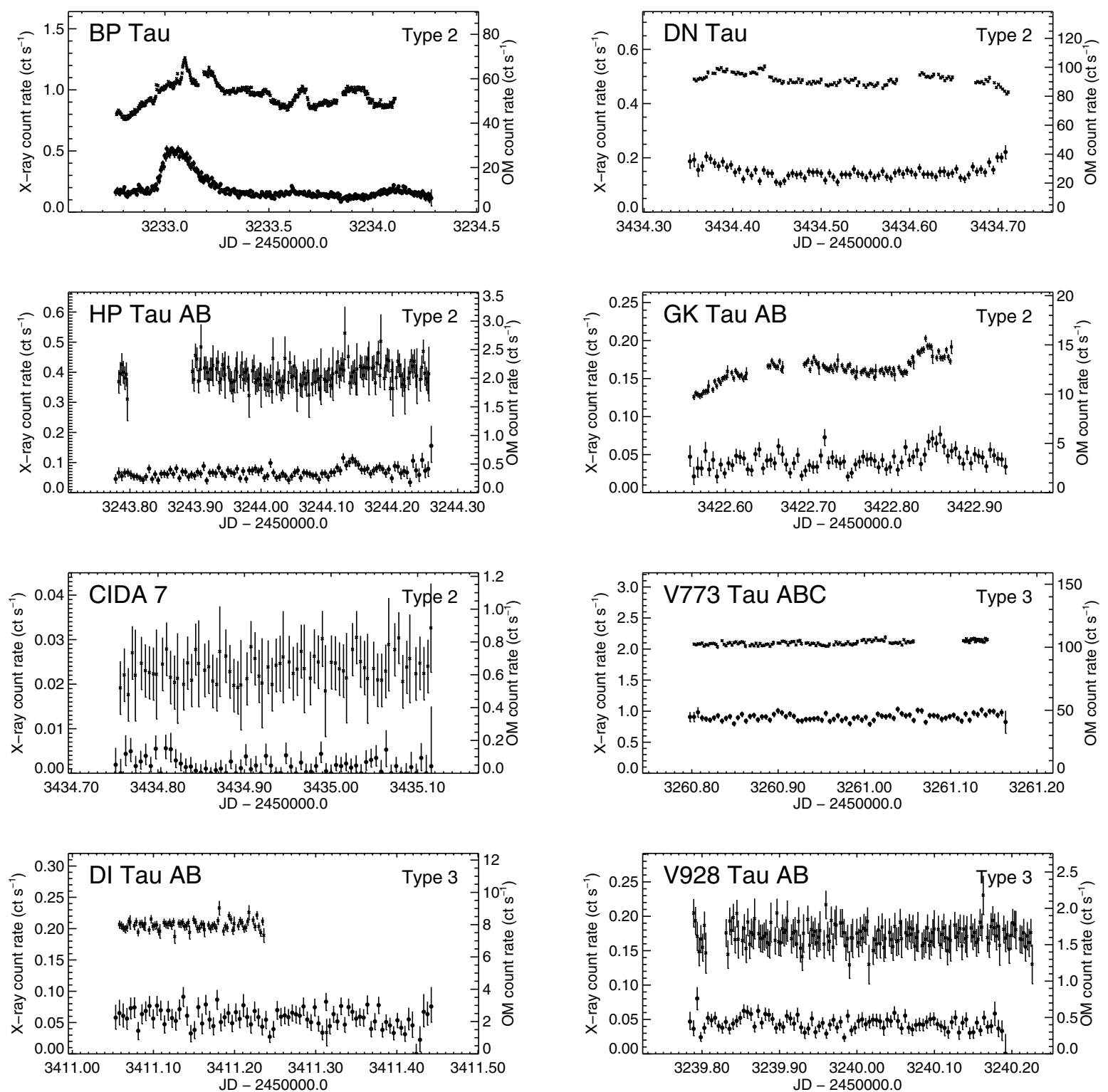

Fig. 8. The TMC sample of stars with OM FAST mode data. The upper curve in each panel is the OM light curve (right $y$-axis), whereas the lower curve is the X-ray light curve (left $y$-axis). The OM light curves were rebinned to bin sizes of $200 \mathrm{~s}$, except for CIDA 7, which required a bin size of $400 \mathrm{~s}$. The type of the source is given together with the source name. The OM FAST data of IT Tau and UZ Tau EW are not shown since the sources were not properly placed on the small OM FAST window, and thus the light curves were unreliable. In addition, the T Tau OM FAST light curve is the subject of a separate paper and will be presented elsewhere (Güdel et al. 2007c). Note that small manual corrections were necessary for DN Tau, DI Tau AB, and BP Tau (see footnote 5).

are not reliable. We also applied some manual corrections to the OM FAST light curves of DN Tau, DI Tau, and BP Tau ${ }^{5}$.

${ }^{5}$ In the case of DN Tau, due to a feature in SAS 6.x, about half the counts were processed in the exposure S009 (DATE-OBS = "2005-0305T01:37:35" and DATE-END = "2005-03-05T02:02:35") only. The average count rate in this exposure is $37.7 \mathrm{ct} \mathrm{s}^{-1}$, after correction for deadtime, aperture, and coincidence losses. The detected count rate for S009 is $32.2 \mathrm{ct} \mathrm{s}^{-1}$ (i.e., 1.17 times smaller), based on the theoretical correction in the XMM-Newton Users Handbook (the empirical correction is minimal). In contrast, the average count rate in the preceding exposure (S408) is $87.7 \mathrm{ct} \mathrm{s}^{-1}$. The latter corresponds to a detected count rate of $63.4 \mathrm{ct} \mathrm{s}^{-1}$ (i.e., 1.38 times smaller). Thus, we multiplied the count rates in S009 by $2 / 1.17 \times 1.38=2.36$ to correct for the SAS feature. For DI Tau, the pointing of the satellite changed unexpectedly after DATE-OBS = "2005-02-09T19:30:13" (exposures S412, S009,
Focusing on the OM FAST light curves, it is clear that type 3 stars show almost no variability in the OM, in contrast to type 2 stars. The classical T Tau star BP Tau shows significant UV variability (the BP Tau data were originally presented

S413-S416), and DI Tau fell just on the edge of the OM FAST window. We thus discarded count rates after this epoch. For BP Tau, a problem with the time stamps of exposure S051 occurred. The DATE-OBS and DATE-END keywords of the following exposure, S052, were incorrectly used, and thus the time stamps of events were incorrectly calculated by the SAS software. We manually corrected these time stamps and keywords by using the correct keywords, DATE-OBS = "2004-0816T06:45:56" DATE-END = "2004-08-16T08:27:51", and incidentally shifting the time stamps by $-6256 \mathrm{~s}$. The same timing problem for the S051 IMAGING data of BP Tau needed a similar correction of the date keywords. 


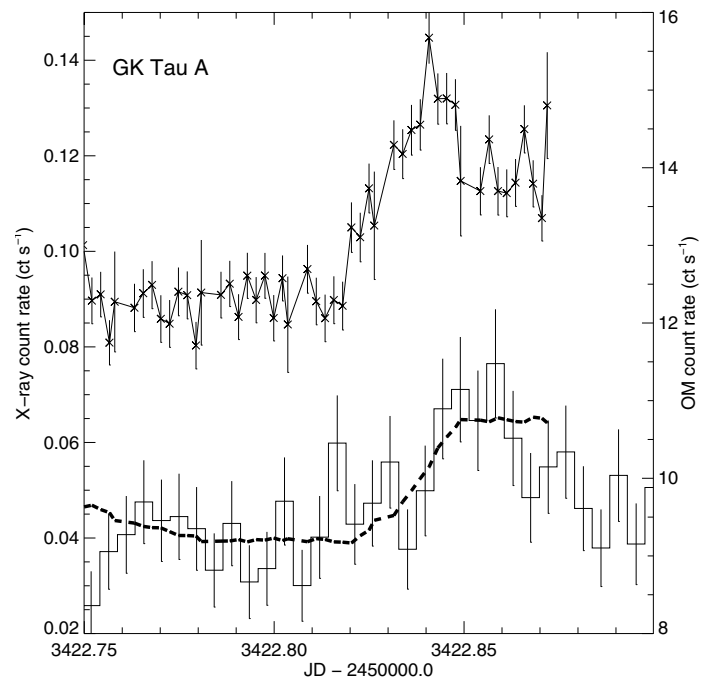

Fig. 9. Details of the GK Tau OM and X-ray light curves. The OM light curve (top curve) was rebinned to a bin size of $200 \mathrm{~s}$, while the Xray light curve (bottom histogram) used a $400 \mathrm{~s}$ bin size. The thick dashed line corresponds to the OM light curve convolved through a kernel $K\left(t, t^{\prime}\right)$ (see text) but it was shifted down for clarity. The GK Tau A event shows evidence of a Neupert-like effect.

by Schmitt et al. 2005). One large X-ray flare is visible at the start of the observation, with two slowly varying flux enhancements that could be due to flares, but also rotational modulation of bright active regions. The $U V W 1$ light curve of BP Tau does not show direct evidence of correlations with the X-ray light curve. In fact, the UV flux peaks after the X-ray light curve, in contradiction with the Neupert effect paradigm. However, such a peculiar behavior is not uncommon in some flares observed in the Sun or in main-sequence or evolved magnetically active stars with no accretion (e.g., Ayres et al. 2001; Stelzer et al. 2003; Osten et al. 2005). Nevertheless, the strong variability in the OM light curve is generally uncorrelated with the X-ray light curve, which strongly suggests that the OM light curve is completely dominated by the UV emission of accretion spots in BP Tau. The period of BP Tau being $7.60 \mathrm{~d}$, much longer than the 1.5 d duration of the observation or typical OM "events" $(0.1-$ $0.2 \mathrm{~d}$ ), it is more probable that such events are due to accretion events such as those observed in the brown dwarf 2MASS J04141188+2811535 (Grosso et al. 2007a).

The GK Tau light curves deserve special attention. Indeed, the OM light curve shows evidence of two behaviors: a general slow variation uncorrelated with the X-ray light curve that is probably due to the rotational modulation of accretion spots $(P=4.60 \mathrm{~d})$, and a short-duration event around JD 2453422.84 that appears related to a small flare-like event in the X-rays. Figure 9 shows an extract of the light curves. Note that the OM emission is from GK Tau A. The companion at 2".5 was not detected in the OM. In the X-rays, the binary cannot be resolved; nevertheless, the A component should contribute the most to the $\mathrm{X}$-ray emission. In addition, the X-ray emission is blended with the X-ray emission of the nearby GI Tau. The X-ray light curves of GK Tau AB and GI Tau shown in this paper take into account this blend since they were extracted from smaller radii (160 pix, i.e., $\left.8^{\prime \prime}\right)$. We convolved the OM light curve through the kernel function, $K\left(t, t^{\prime}\right)$, where $K\left(t, t^{\prime}\right)=\mathrm{e}^{-\left(t-t^{\prime}\right) / \tau}$ if $t^{\prime}<t$ and $K\left(t, t^{\prime}\right)=0$ otherwise, and where $\tau$ was set to $2 \mathrm{ks}$; for clarity and comparison with the X-ray light curve, we introduced a vertical offset to the convolved light curve. The convolution of the
OM light curve is similar to an integration; however, the use of the exponential function in the kernel allows us to mimic the decaying effect of radiative and conductive cooling in X-rays. This exercise is a good indication that the event observed in X-rays and in the OM in GK Tau is a flare that displayed a Neupertlike effect, with the optical emission peaking before the X-rays. A similar case is reported in DG Tau A with OM IMAGING data (Güdel et al. 2007b). GK Tau's OM light curve can, therefore, be seen as a combination of stellar photospheric emission, accretion spot emission producing slow variability, and flare-like events. As seen from GK Tau, but most visibly BP Tau (and other type 2 stars), the long-term variability due to accretion does not appear to correlate with the X-ray variability in general. This indicates that the contribution of accretion to the $\mathrm{X}$-ray emission is limited, probably to the soft component only, as suggested from high-resolution X-ray spectra of classical T Tau stars (Telleschi et al. 2007a). In contrast, type 3 stars with little or no accretion do not show evidence of slow variability in the OM.

\section{Summary and conclusions}

We have presented results from the Optical Monitor data of the $X M M-N e w t o n$ Extended Survey of the Taurus Molecular Cloud. Optical $(U)$ or ultraviolet $(U V W 1$ or $U V W 2)$ magnitudes were obtained; in addition low time resolution light curves from the IMAGING data were compiled and compared to the X-ray light curves. For a few sources, FAST mode data with high time resolution were available as well.

The OM data are unique since they provide strictly simultaneous coverage in the optical (or ultraviolet) together with $\mathrm{X}$-rays. This XMM-Newton capability allowed us to study light curves; we were able to detect Neupert-like effects in the accreting stars GK Tau A and DG Tau A, but also slow variability in accreting stars that is most likely due to accretion spots. A statistical analysis shows that, although observed $U$ magnitudes are similar in type 2 and 3 stars, accreting stars are, in fact, brighter in the $U$ band than type 3 stars after correction for extinction. This excess emission most likely originates from accretion. This behavior, although not a new discovery, is now shown to be disconnected from the X-ray variability. Indeed, for example, the light curve of BP Tau shows strong ultraviolet variability that is not reproduced in the X-ray light curve. In contrast, nonaccreting stars (or stars with low accretion rates) do not show evidence of slow variability. Their emission is therefore probably dominated by photospheric emission. Similar results were found in the optical $(B V R I)$ and X-ray data of Orion Nebula Cluster pre-main sequence stars (Stassun et al. 2006).

The XEST OM catalogue lists 2148 detected sources and about $88 \%$ (1893) OM sources had 2MASS counterparts; in contrast, 916 sources were detected in the X-rays and had coordinates such that they could be detected in the OM (not necessarily). However, only $98 \mathrm{X}$-ray sources matched OM sources, after astrometric corrections applied to both the X-rays and the OM coordinates based on 2MASS coordinates. Of these 98 sources, only 51 are considered as TMC members (12 are bona-fide TMC candidates; Scelsi et al. 2007). Therefore, the vast majority (about 97\%) detected in the OM images are, in fact, not TMC members. While it is hard to determine the origin of a target based on X-ray or optical data alone, the OM data, together with 2MASS and Spitzer data (Padgett et al. 2006, in preparation), should provide useful constraints to discriminate between stellar and non-stellar objects, and thus discover new members of the Taurus Molecular Cloud. 
Acknowledgements. We thank the anonymous referee for detailed comments and suggestions that helped to improve this manuscript. M. A. acknowledges support by National Aeronautics and Space Administration (NASA) grant NNG05GF92G and from a Swiss National Science Foundation Professorship (PP002-110504). L. S. ackoweldges financial contribution from contract ASIINAF I/023/05/0 and M.I.U.R. We acknowledge financial support by the International Space Science Institute (ISSI) in Bern to the XMM-Newton XEST team. The PSI group was supported by the Swiss National Science Foundation (grants 20-66875.01 and 20-109255/1). Part of this research is based on observations obtained with XMM-Newton, an ESA science mission with instruments and contributions directly funded by ESA member states and the USA (NASA). This publication makes use of data products from the Two Micron All Sky Survey (2MASS), which is a joint project of the University of Massachusetts and the Infrared Processing and Analysis Center/California Institute of Technology, funded by NASA and the National Science Foundation. Our research made use of the SIMBAD database, operated at CDS, Strasbourg, France.

\section{References}

Antonucci, E., Gabriel, A. H., \& Dennis, B. R. 1984, ApJ, 287, 917 Audard, M., Güdel, M., Skinner, S. L., et al. 2005, ApJ, 635, L81

Ayres, T. R., Brown, A., Osten, R. A., et al. 2001, ApJ, 549, 554

Bouvier, J. 1990, AJ, 99, 946

Bouvier, J., \& Bertout, C. 1989, A\&A, 211, 99

Bouvier, J., Bertout, C., \& Bouchet, P. 1988, A\&AS, 75, 1

Bouvier, J., Cabrit, S., Fernandez, M., Martin, E. L., \& Matthews, J. M. 1993, A\&A, 272, 176

Bouvier, J., Covino, E., Kovo, O., et al. 1995, A\&A, 299, 89

Bouvier, J., Wichmann, R., Grankin, K., et al. 1997, A\&A, 318, 495

Cardelli, J. A., Clayton, G. C., \& Mathis, J. S. 1989, ApJ, 345, 245

Ehle, M., et al. 2005, XMM-Newton Users' Handbook (v2.3)

Flaccomio, E., Micela, G., Sciortino, S., et al. 2005, ApJS, 160, 450

Grosso, N., Kastner, J. H., Ozawa, et al. 2005, A\&A, 438, 159

Grosso, N., Audard, M., Bouvier, J., Briggs, K. R., \& Güdel, M. 2007a, A\&A, 468,557

Grosso, N., Briggs, K. R., Güdel, M., et al. 2007b, A\&A, 468, 391

Güdel, M., Benz, A. O., Schmitt, J. H. M. M., \& Skinner, S. L. 1996, ApJ, 471, 1002

Güdel, M., Audard, M., Skinner, S. L., \& Horvath, M. I. 2002a, ApJ, 580, L73

Güdel, M., Audard, M., Smith, K. W., et al. 2002b, ApJ, 577, 371

Güdel, M., Audard, M., Reale, F., Skinner, S. L., \& Linsky, J. L. 2004, A\&A, 416, 713

Güdel, M., Skinner, S. L., Briggs, K. R., et al. 2005, ApJ, 626, L53

Güdel, M., Briggs, K. R., Arzner, K., et al. 2007a, A\&A, 468, 353
Güdel, M., Telleschi, A., Audard, M., et al. 2007b, A\&A, 468, 515

Güdel, M., Skinner, S. L., Mel'nikov, S. Y., et al. 2007c, A\&A, 468, 529

Hawley, S. L., Fisher, G. H., Simon, T., et al. 1995, ApJ, 453, 464

Hawley, S. L., Allerd, J. C., Johns-Krull, C. M., et al. 2003, ApJ, 597, 535

Herbst, W., Herbst, D. K., Grossman, E. J., \& Weinstein, D. 1994, AJ, 108, 1906

den Herder, J. W., Brinkman, A. C., Kahn, S. M., et al. 2001, A\&A, 365, L7

Jansen, F., Lumb, D., Altieri, B., et al. 2001, A\&A, 365, L1

Kastner, J. H., Huenemoerder, D. P., Schulz, N. S., Canizares, C. R., \& Weintraub, D. A. 2002, ApJ, 567, 434

Kastner, J. H., Richmond, M., Grosso, N., et al. 2004, Nature, 430, 429

Kastner, J. H., Franz, G., Grosso, N., et al. 2005, ApJS, 160, 511

Kastner, J. H., Richmond, M., Grosso, N., et al. 2006, ApJ, 648, L43

Kenyon, S. J., \& Hartmann, L. 1995, ApJS, 101, 117

Luhman, K. L. 2004, ApJ, 617, 1216

Mason, K. O., Breeveld, A., Much, R., et al. 2001, A\&A, 365, L36

Mitra-Kraev, U., Harra, L. K., Güdel, M., et al. 2005, A\&A, 431, 679

Ness, J.-U., \& Schmitt, J. H. M. M. 2005, A\&A, 444, L41

Neupert, W. M. 1968, ApJ, 153, L59

Osten, R. A., Hawley, S. L., Allred, J. C., Johns-Krull, C. M., \& Roark, C. 2005, ApJ, 621, 398

Robrade, J., \& Schmitt, J. H. M. M. 2006, A\&A, 449, 737

Rydgren, A. E., \& Vrba, F. J. 1983, ApJ, 267, 191

Rydgren, A. E., Zak, D. S., Vrba, F. J., Chugainov, P. F., \& Zajtseva, G. V. 1984, AJ, 89, 1015

Scelsi, L., Maggio, A., Micela, G., et al. 2007, A\&A, 468, 405

Schmitt, J. H. M. M., Robrade, J., Ness, J.-U., Favata, F., \& Stelzer, B. 2005, A\&A, 432, L35

Siess, L., Dufour, E., \& Forestini, M. 2000, A\&A, 358, 593

Smith, K., Güdel, M., \& Audard, M. 2005, A\&A, 436, 241

Stassun, K. G., van den Berg, M., Feigelson, E., \& Flaccomio, E. 2006, ApJ, 649, 914

Stelzer, B., \& Schmitt, J. H. M. M. 2004, A\&A, 418, 687

Stelzer, B., Fernández, M., Costa, V. M., et al. 2003, A\&A, 411, 517

Strüder, L., Briel, U., Dennerl, K., et al. 2001, A\&A, 365, L18

Swartz, D. A., Drake, J. J., Elsner, R. F., et al. 2005, ApJ, 628, 811

Telleschi, A., Güdel, M., Briggs, K. R., Audard, M., \& Scelsi, L. 2007a, A\&A, 468,443

Telleschi, A., Güdel, M., Briggs, K. R., et al. 2007b, A\&A, 468, 541

Turner, M. J. L., Abbey, A., Arnaud, M., et al. 2001, A\&A, 365, L27

Vrba, F. J., Rydgren, A. E., Chugainov, P. F., Shakovskaia, N. I., \& Zak, D. S. 1986, ApJ, 306, 199

Vrba, F. J., Rydgren, A. E., Chugainov, P. F., Shakovskaia, N. I., \& Weaver, W. B. 1989, AJ, 97, 483

Vrba, F. J., Chugainov, P. F., Weaver, W. B., \& Stauffer, J. S. 1993, AJ, 106, 1608 
M. Audard et al.: XMM-Newton OM survey of the TMC, Online Material $p 1$

\section{Online Material}


M. Audard et al.: XMM-Newton OM survey of the TMC, Online Material $p 2$

Appendix B: X-ray and OM light curves
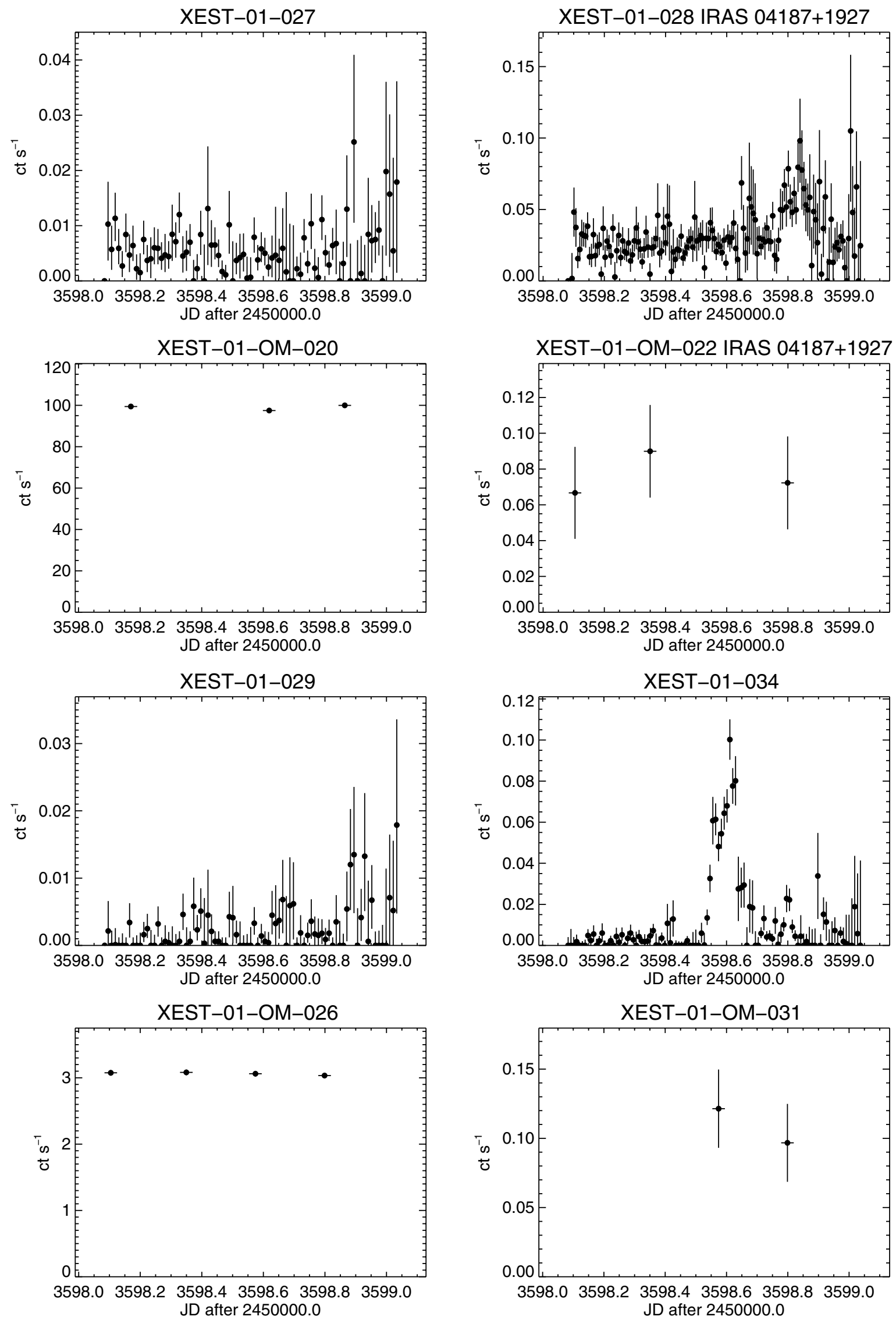

Fig. B.1. Light curves. 
M. Audard et al.: XMM-Newton OM survey of the TMC, Online Material p 3
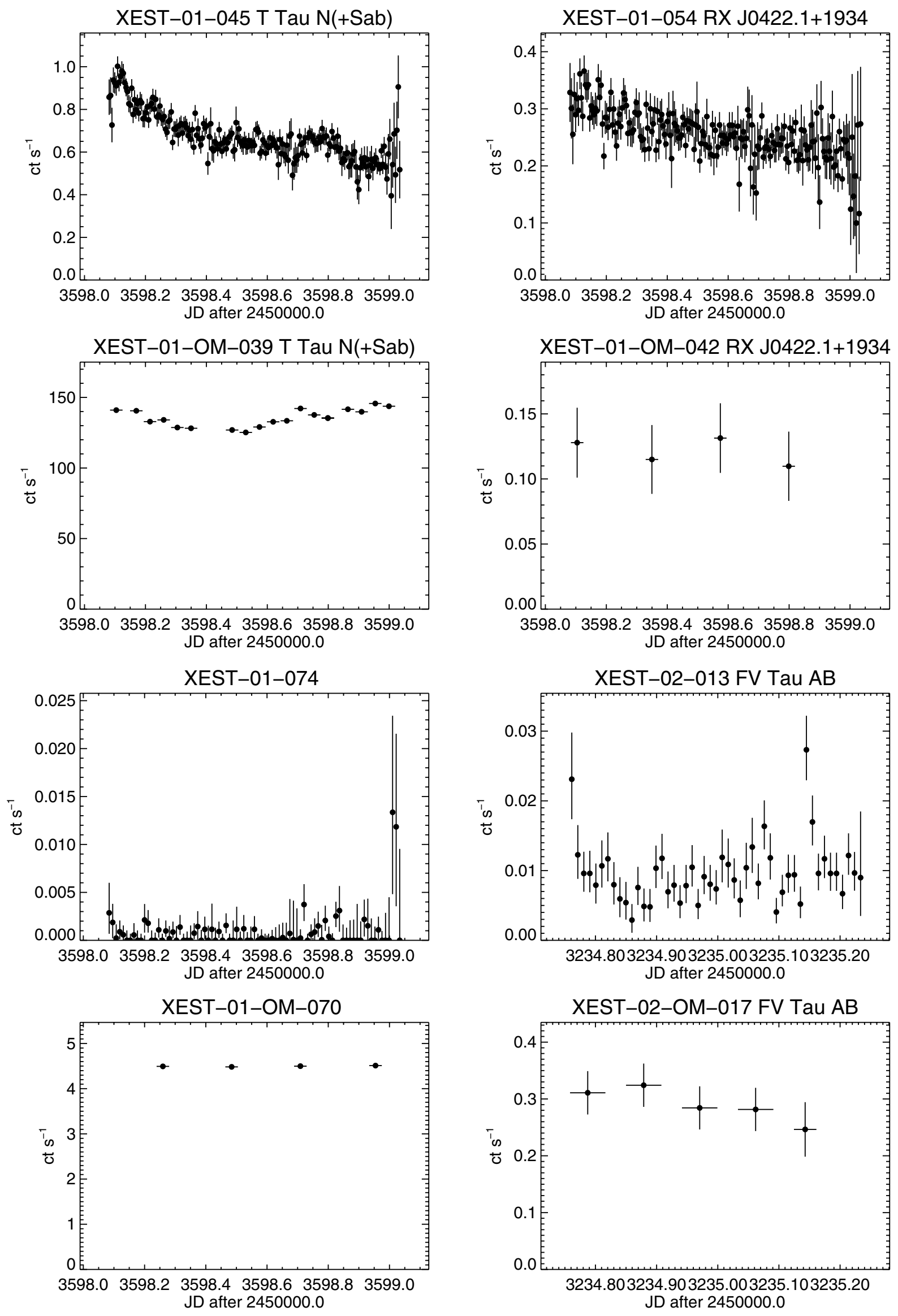

Fig. B.1. continued. 
M. Audard et al.: XMM-Newton OM survey of the TMC, Online Material p 4
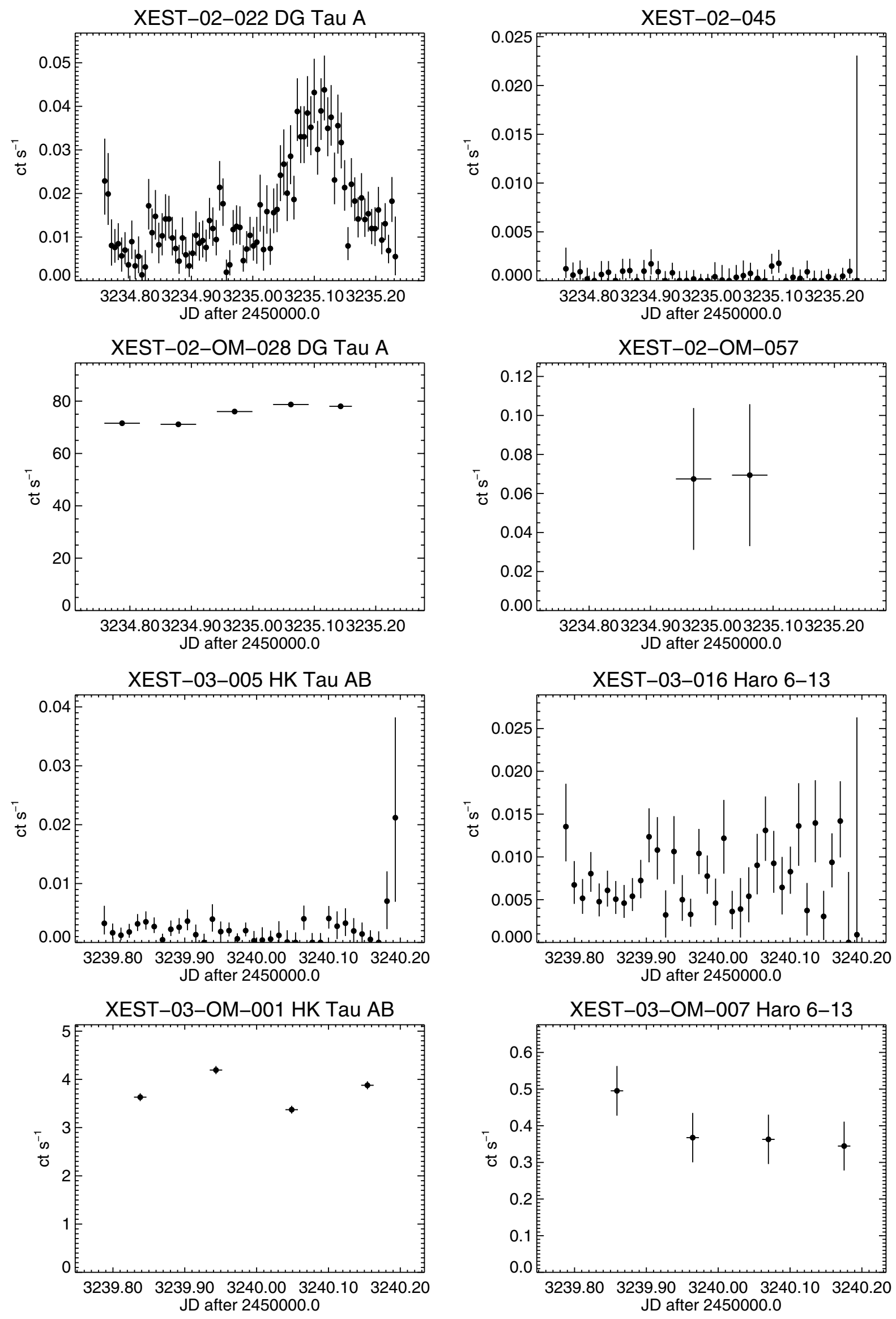

Fig. B.1. continued. 
M. Audard et al.: XMM-Newton OM survey of the TMC, Online Material p 5
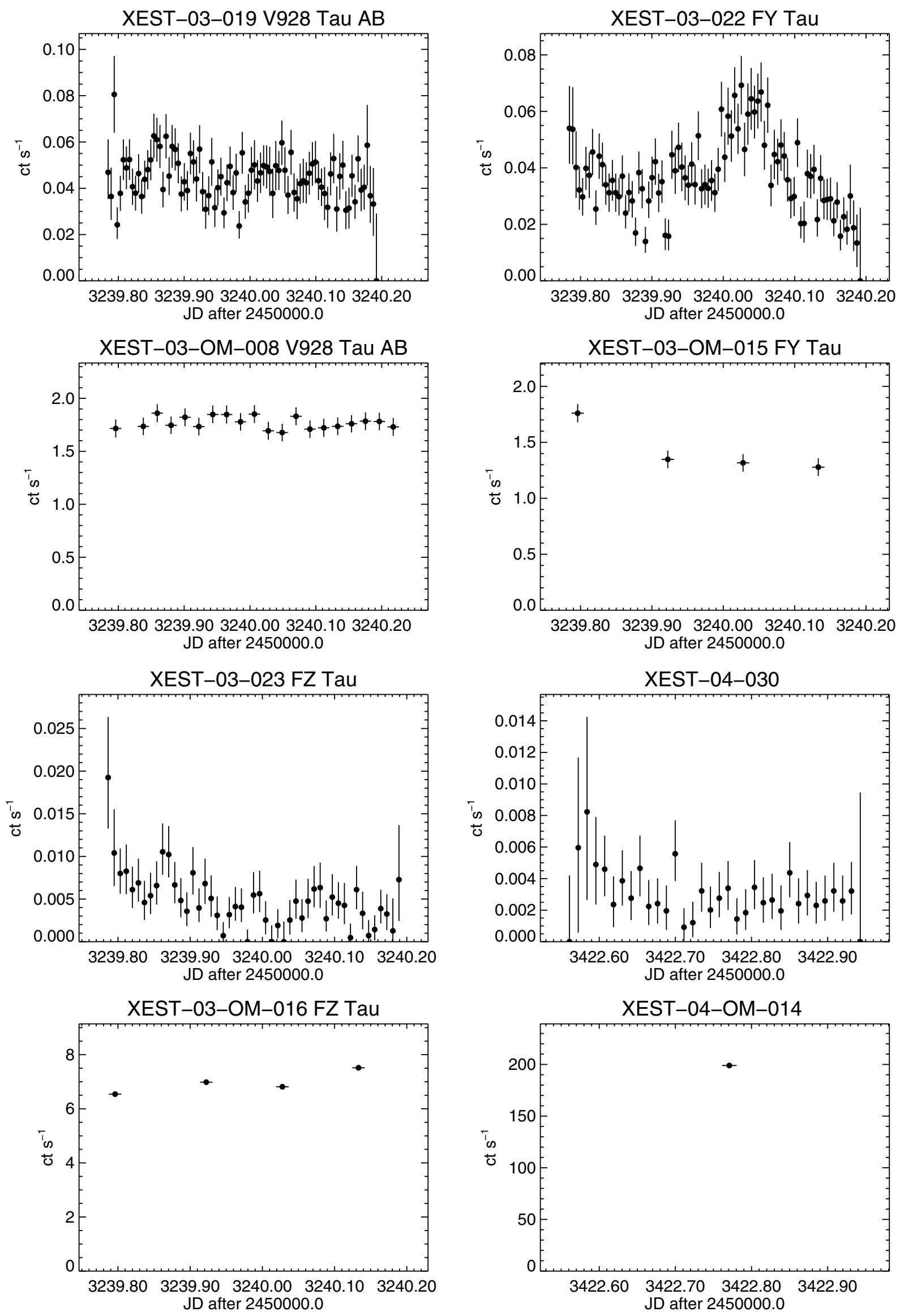

Fig. B.1. continued. 
M. Audard et al.: XMM-Newton OM survey of the TMC, Online Material p 6
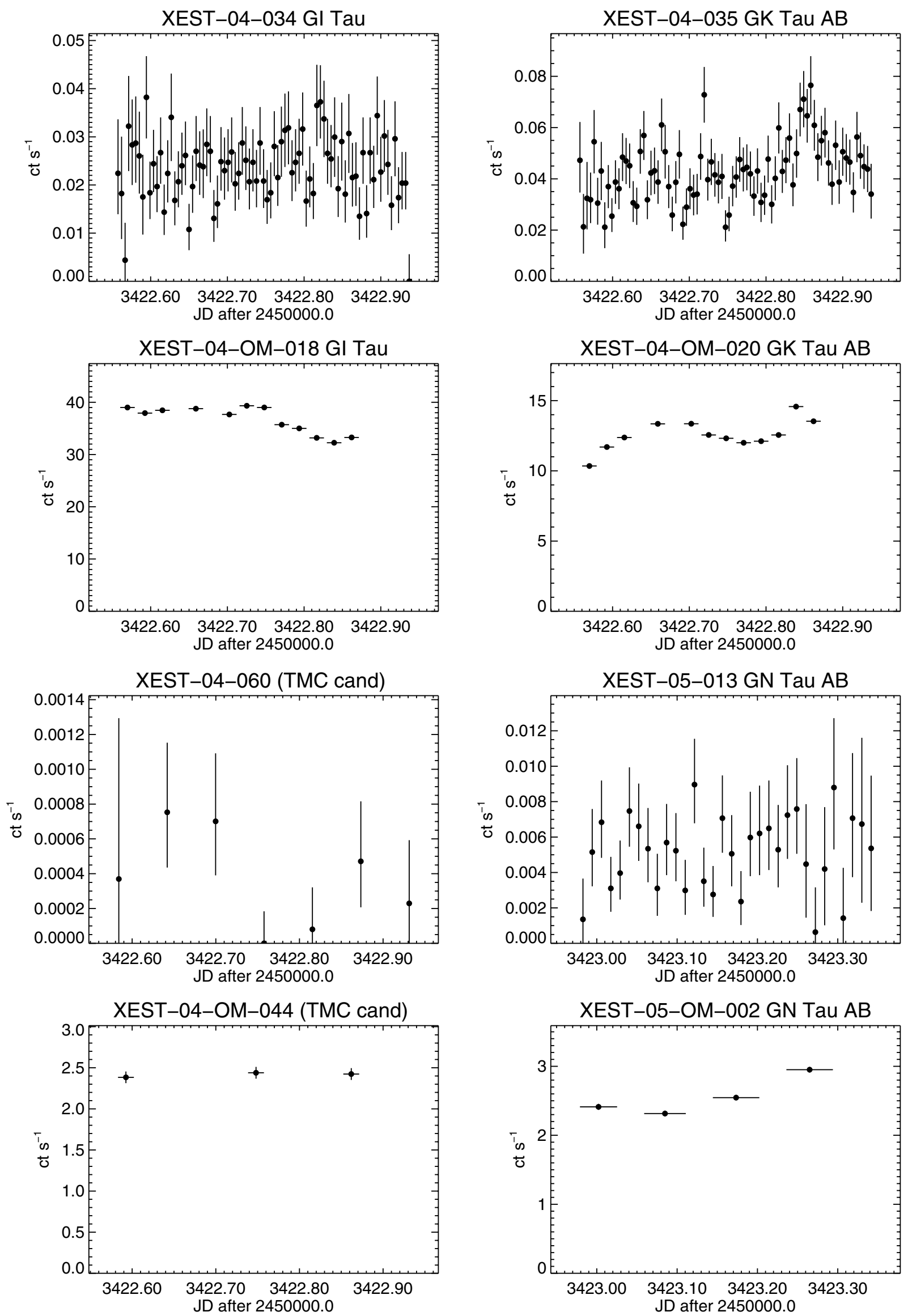

Fig. B.1. continued. 
M. Audard et al.: XMM-Newton OM survey of the TMC, Online Material $p 7$
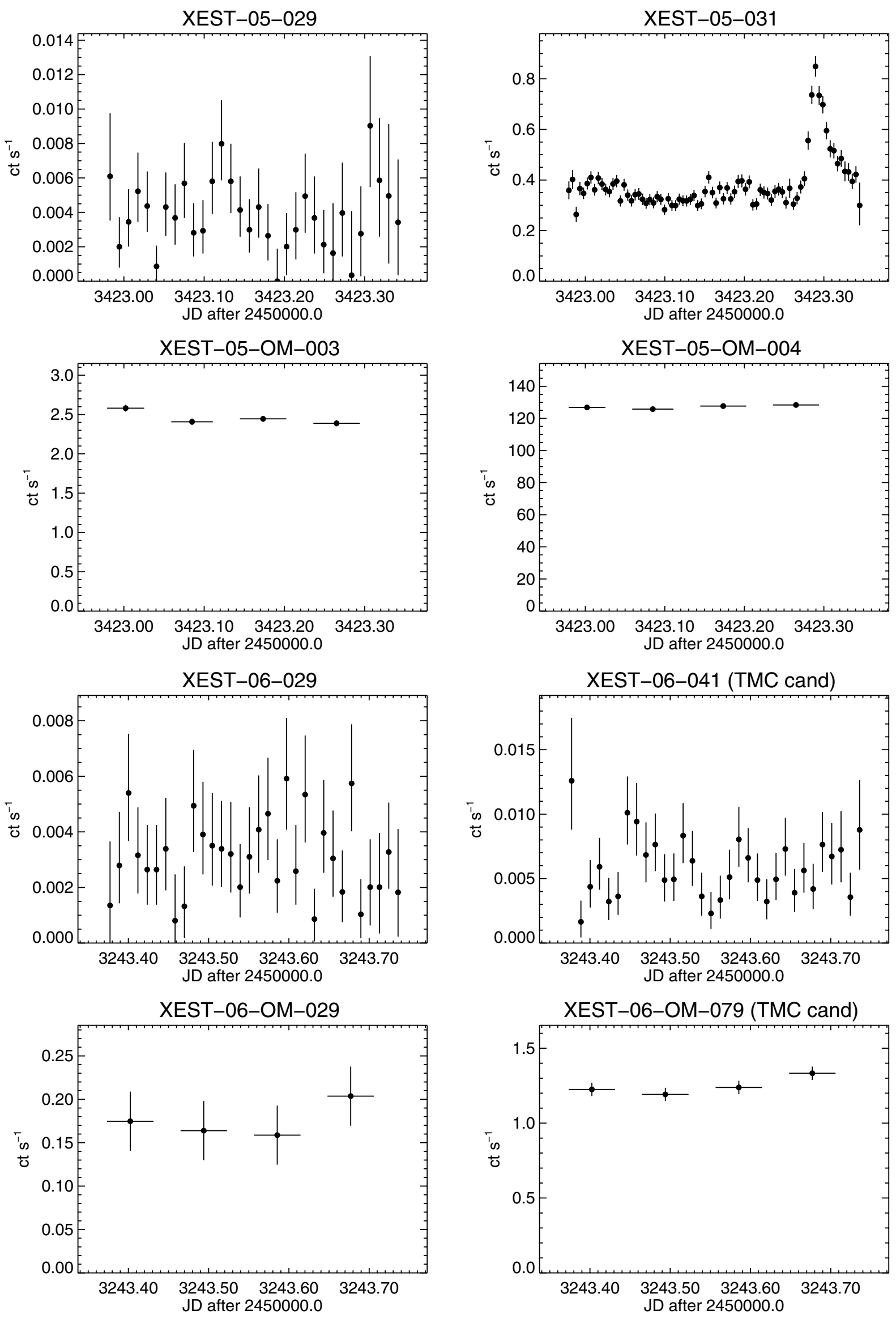

Fig. B.1. continued. 
M. Audard et al.: XMM-Newton OM survey of the TMC, Online Material $p 8$
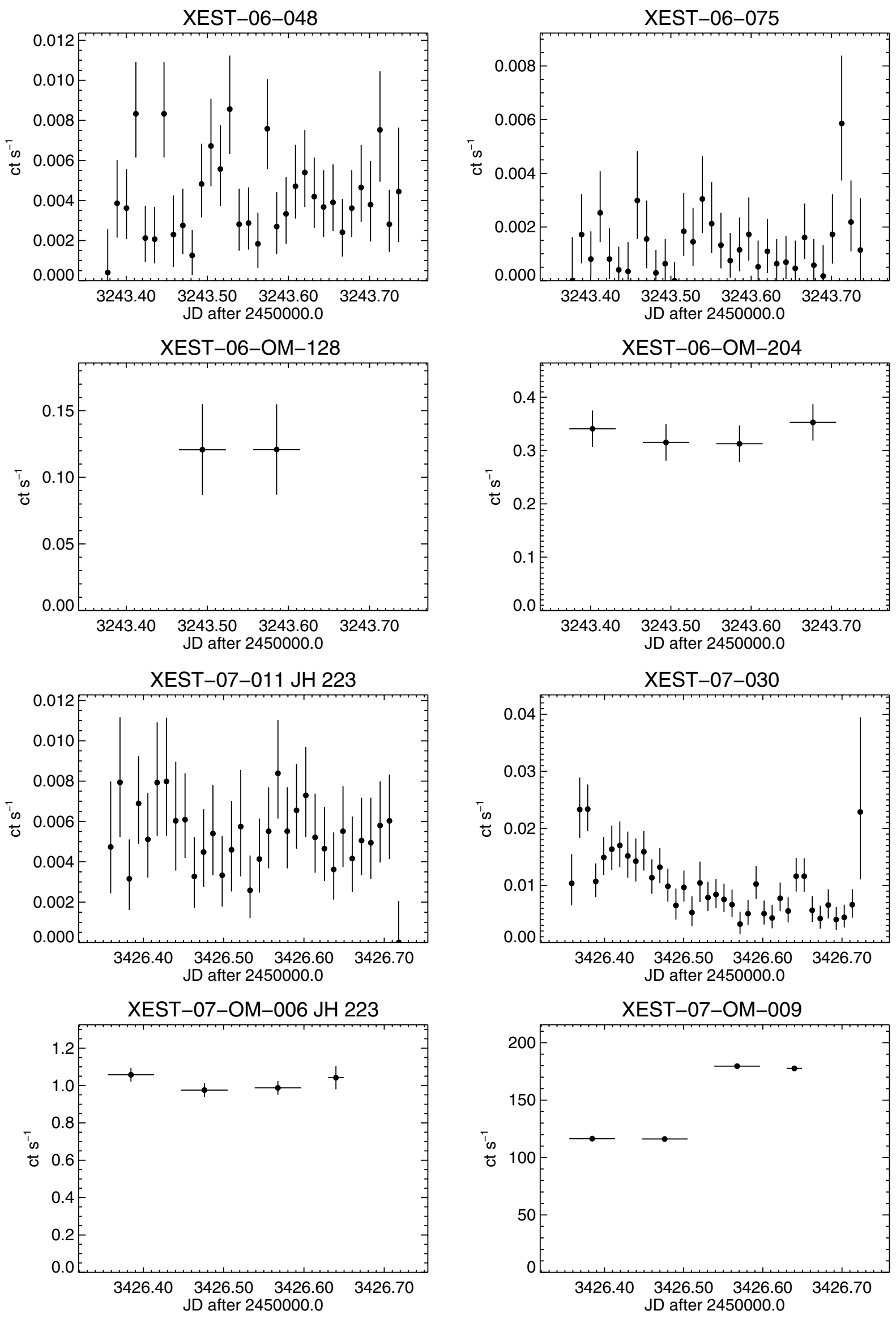

Fig. B.1. continued. 
M. Audard et al.: XMM-Newton OM survey of the TMC, Online Material $p 9$
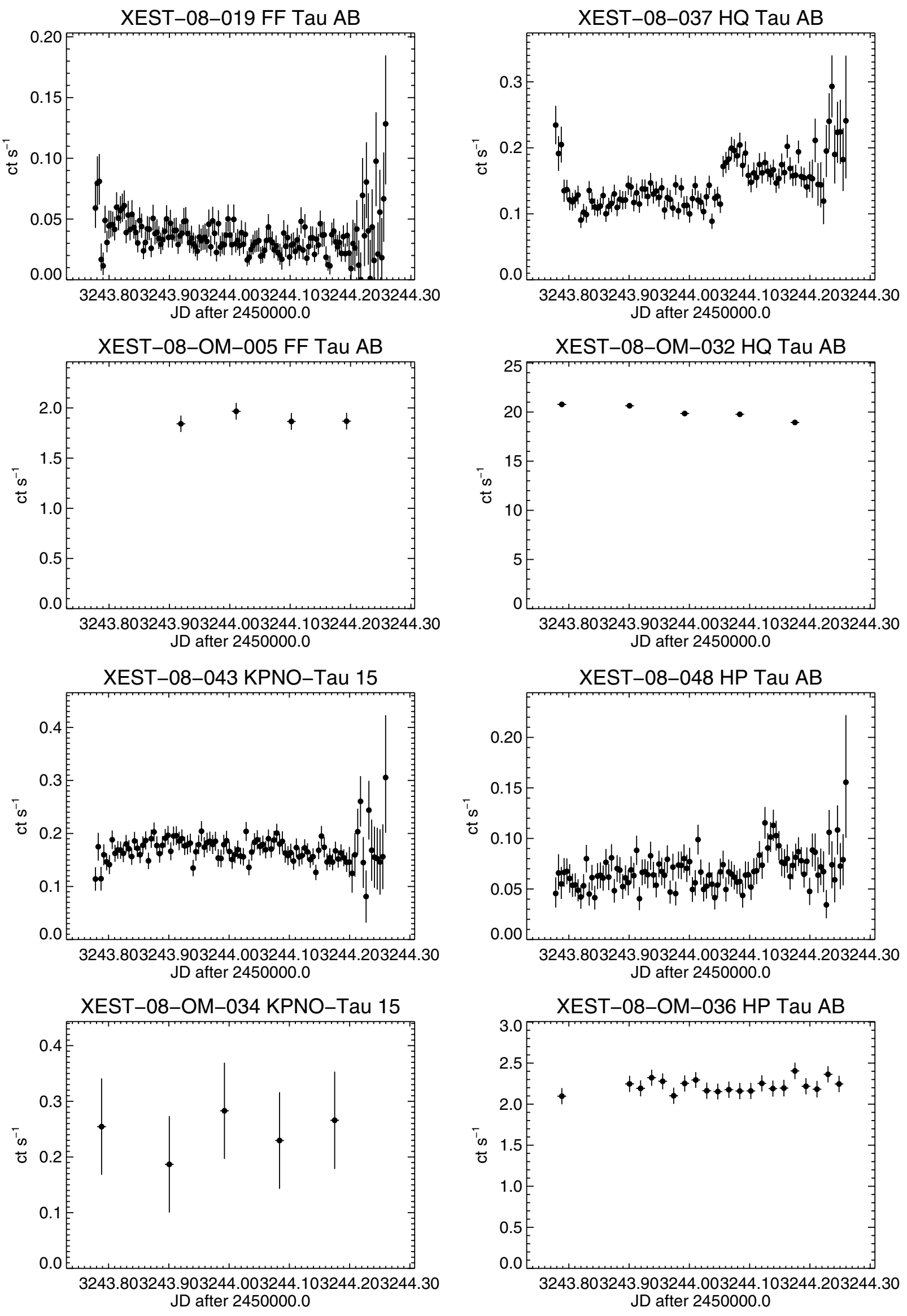

Fig. B.1. continued. 
M. Audard et al.: XMM-Newton OM survey of the TMC, Online Material p 10
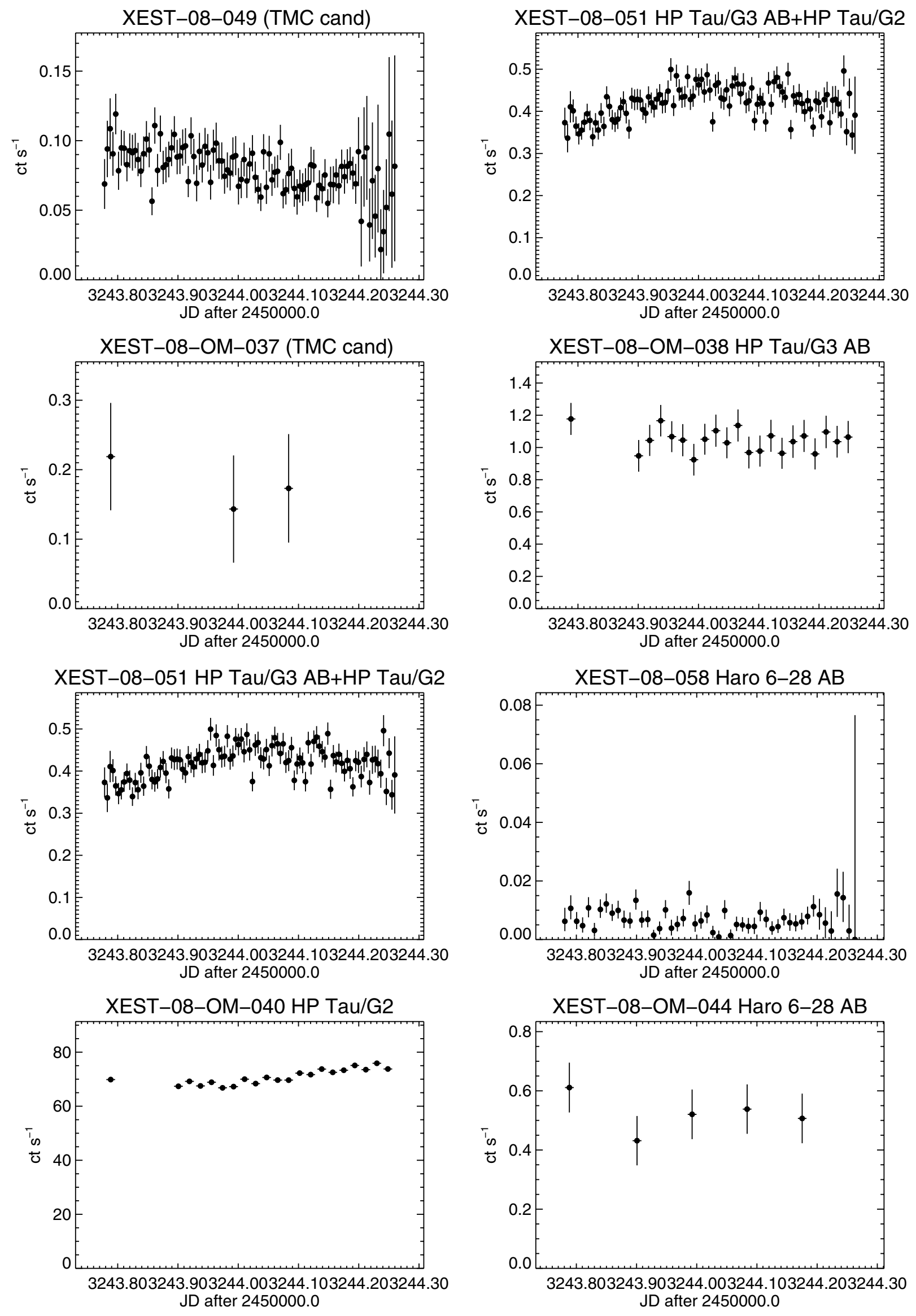

Fig. B.1. continued. 
M. Audard et al.: XMM-Newton OM survey of the TMC, Online Material $p 11$
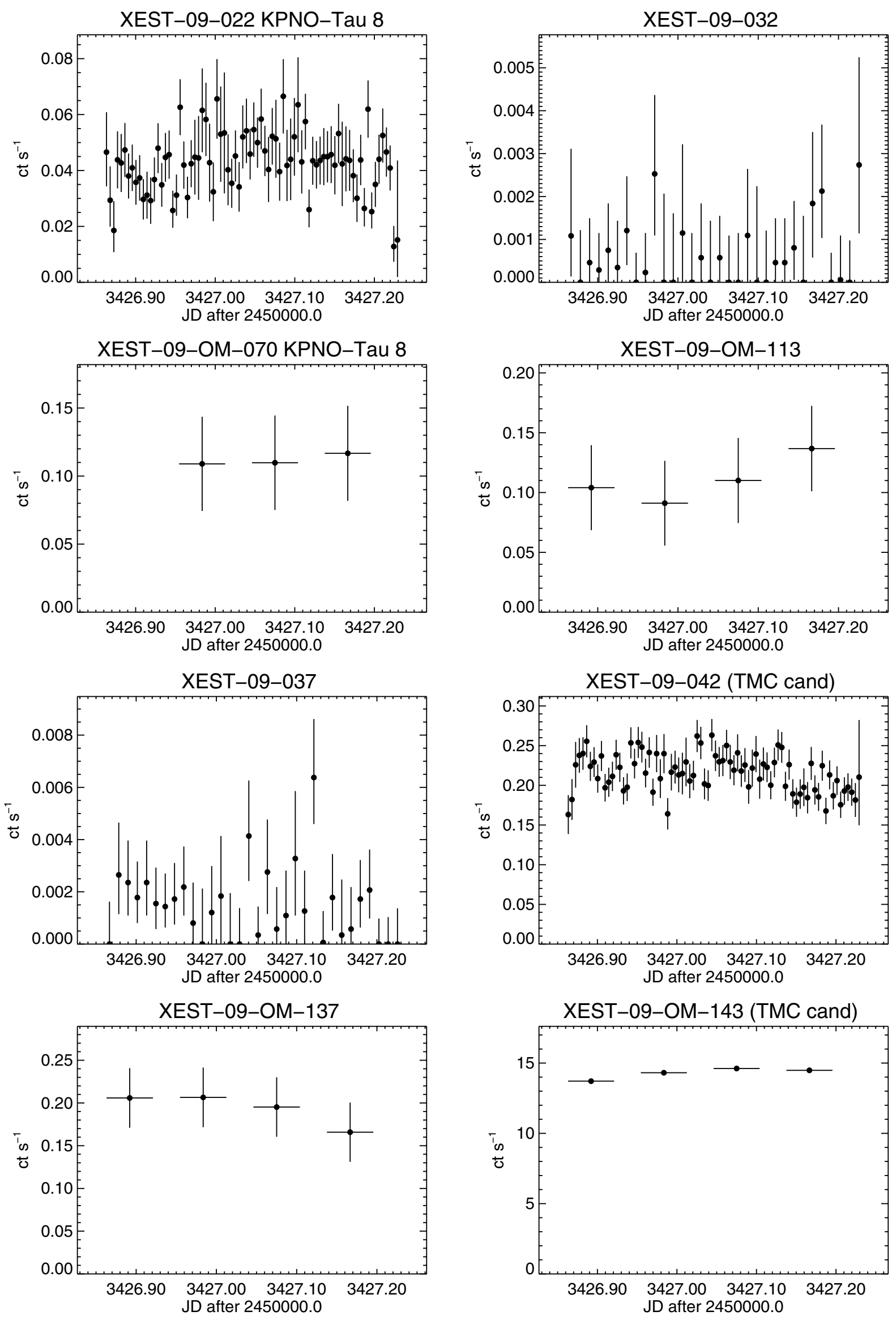

Fig. B.1. continued. 
M. Audard et al.: XMM-Newton OM survey of the TMC, Online Material p 12
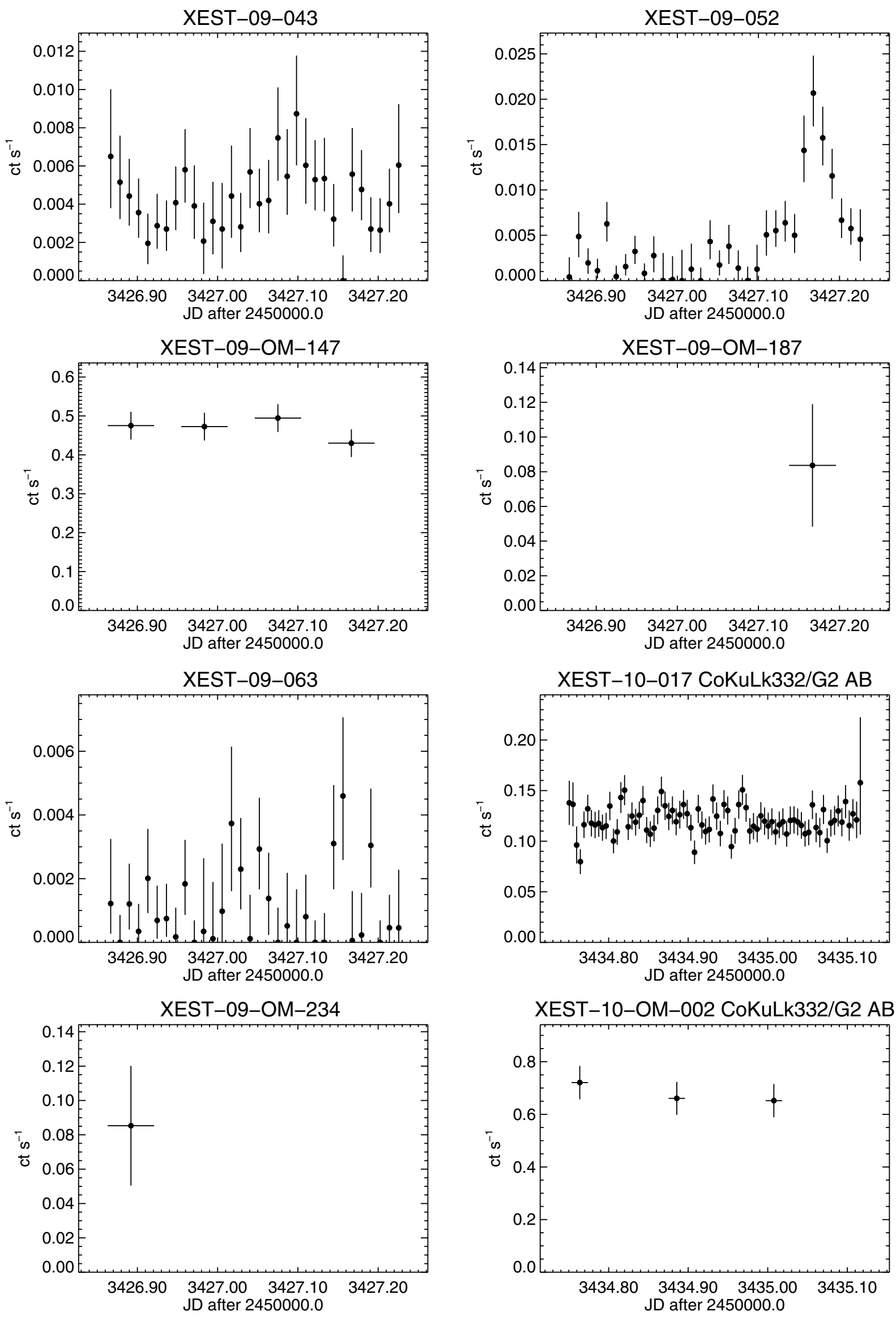

Fig. B.1. continued. 
M. Audard et al.: XMM-Newton OM survey of the TMC, Online Material p 13
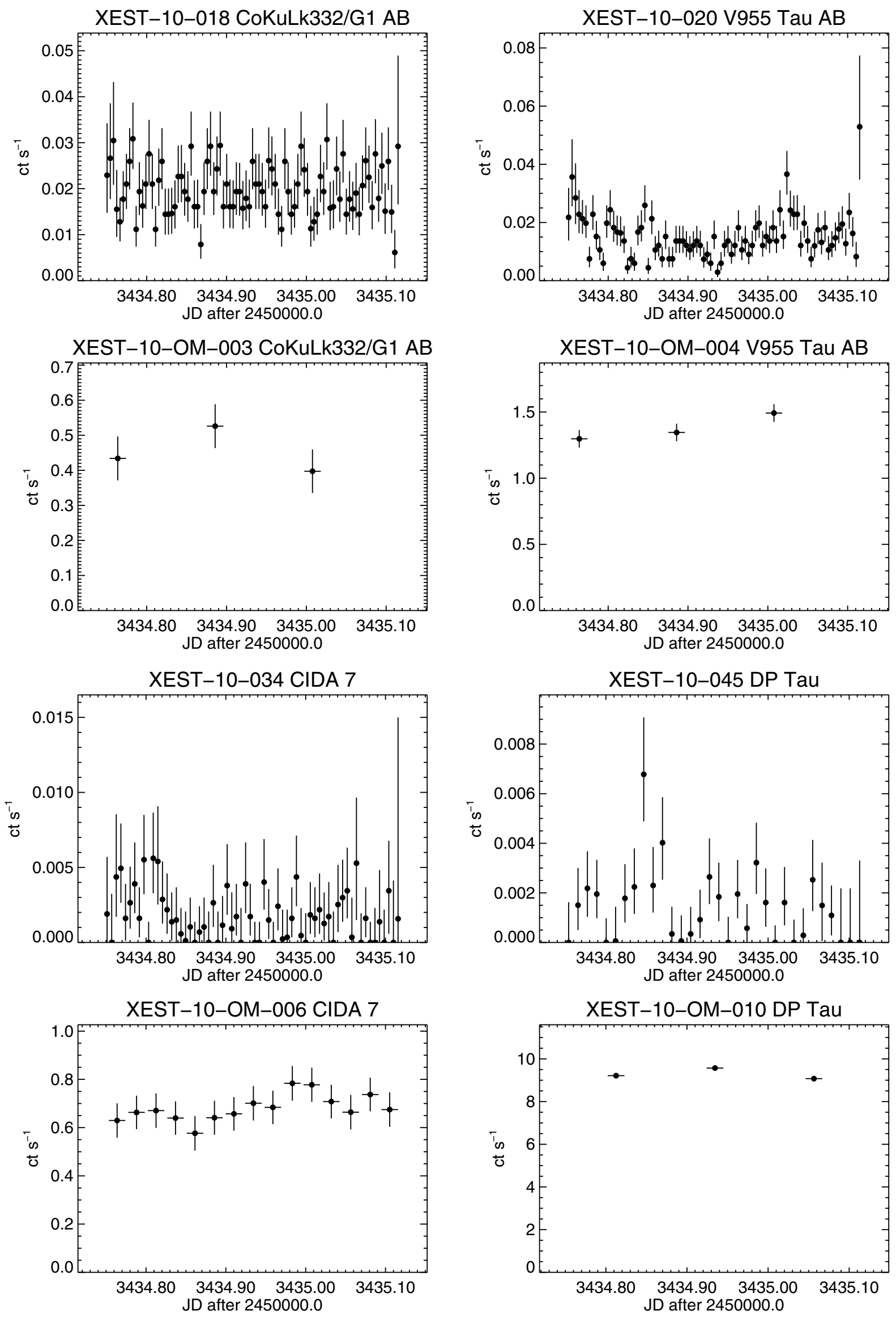

Fig. B.1. continued. 
M. Audard et al.: XMM-Newton OM survey of the TMC, Online Material p 14
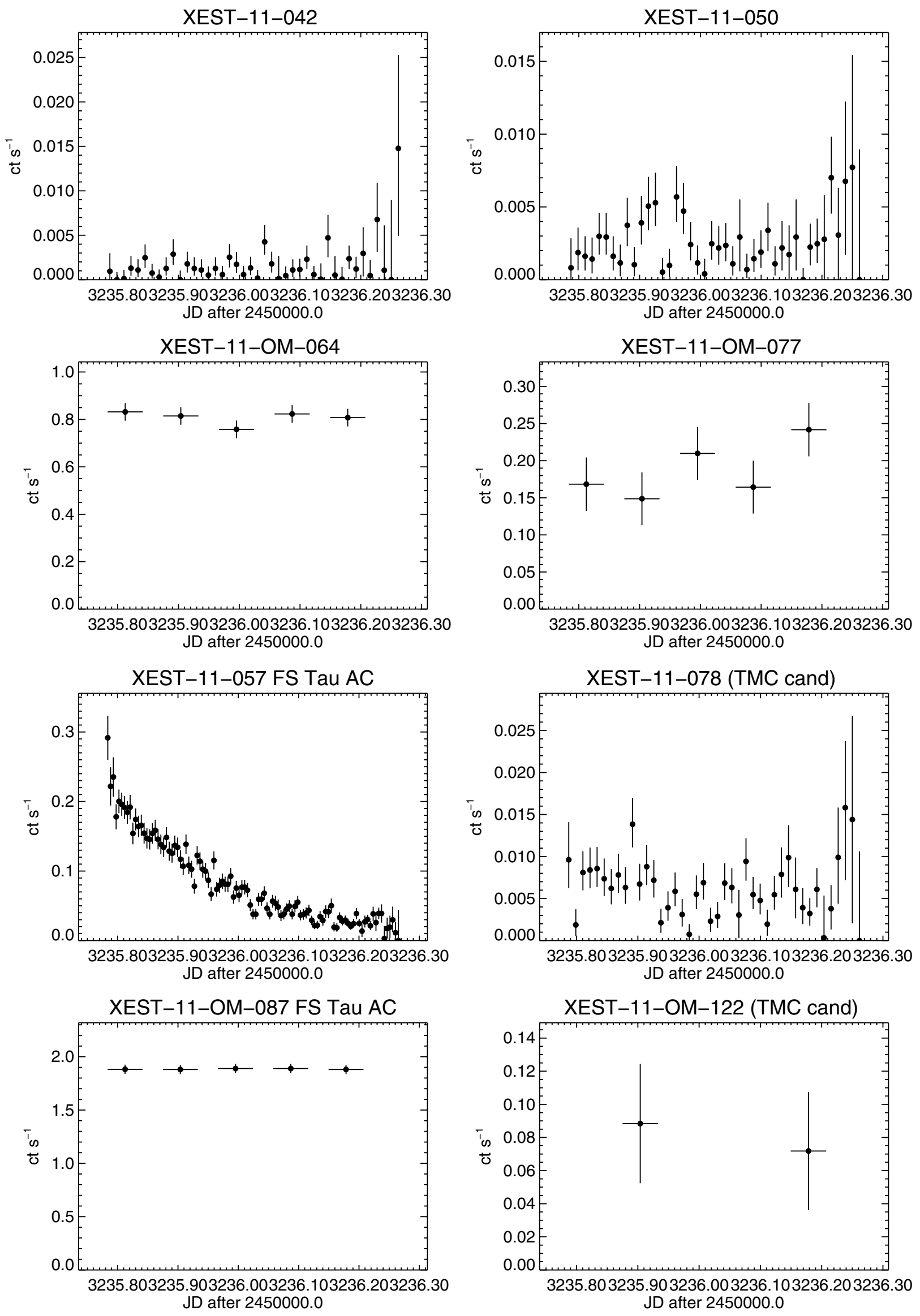

Fig. B.1. continued. 
M. Audard et al.: XMM-Newton OM survey of the TMC, Online Material p 15
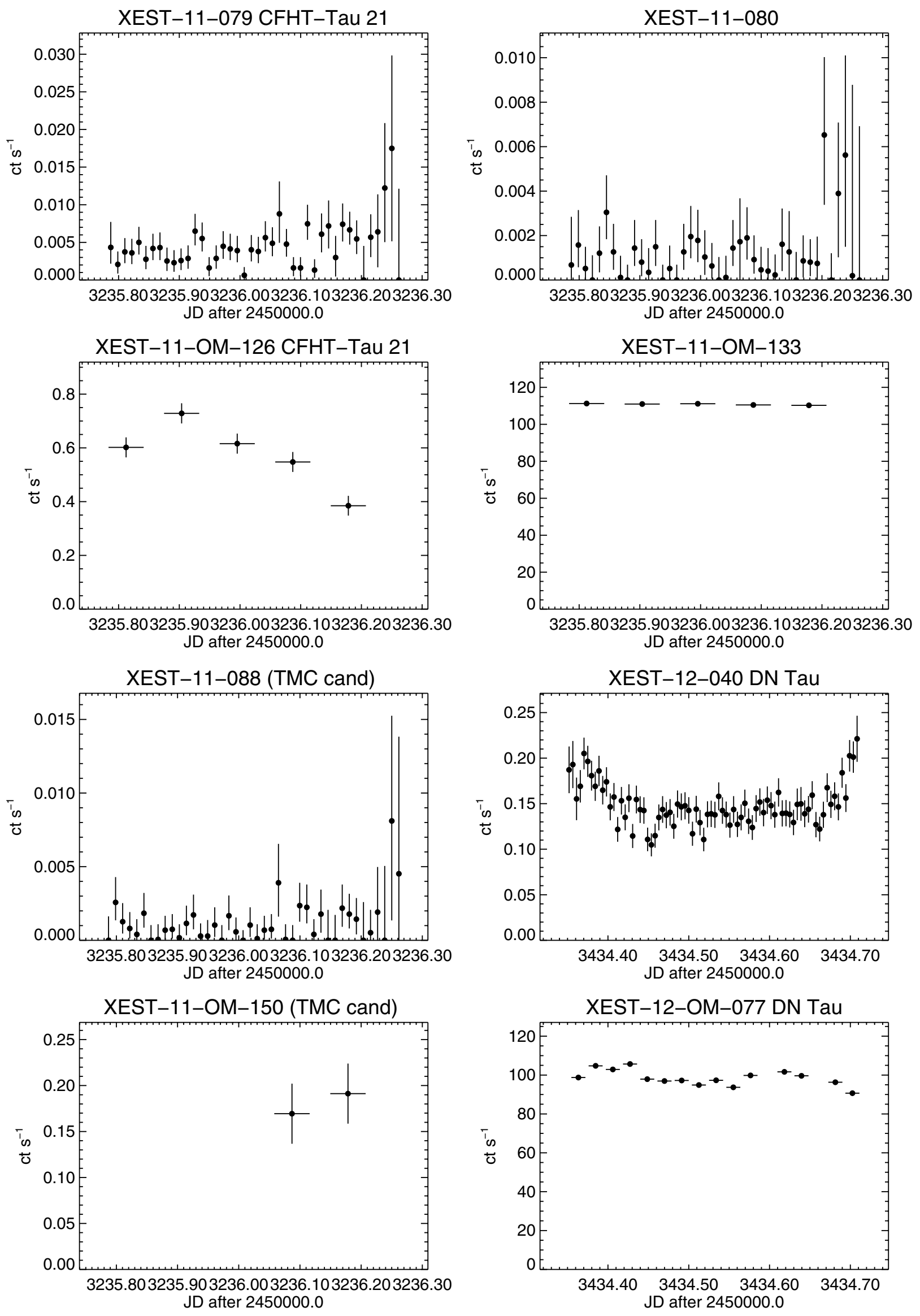

Fig. B.1. continued. 
M. Audard et al.: XMM-Newton OM survey of the TMC, Online Material p 16
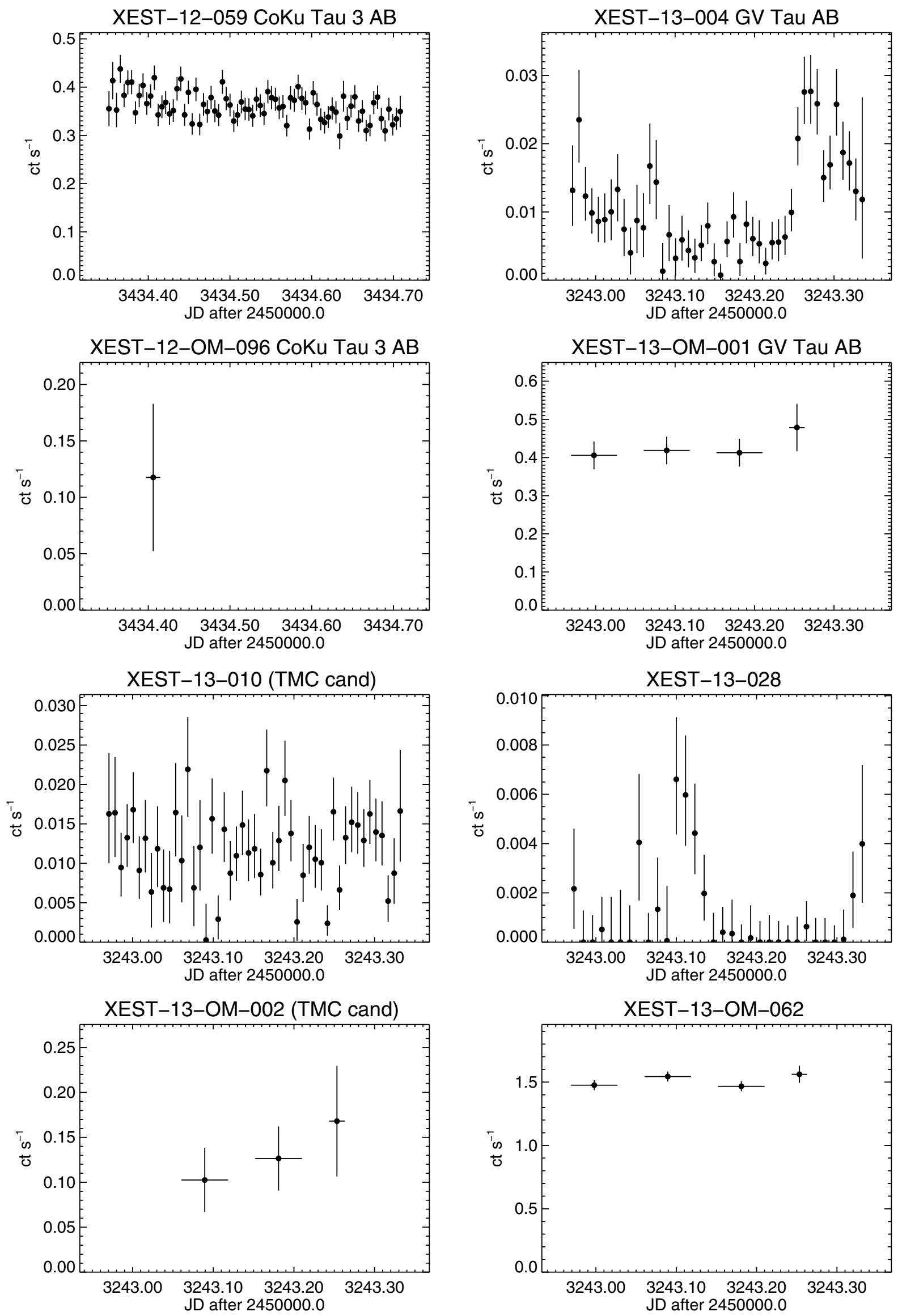

Fig. B.1. continued. 
M. Audard et al.: XMM-Newton OM survey of the TMC, Online Material p 17
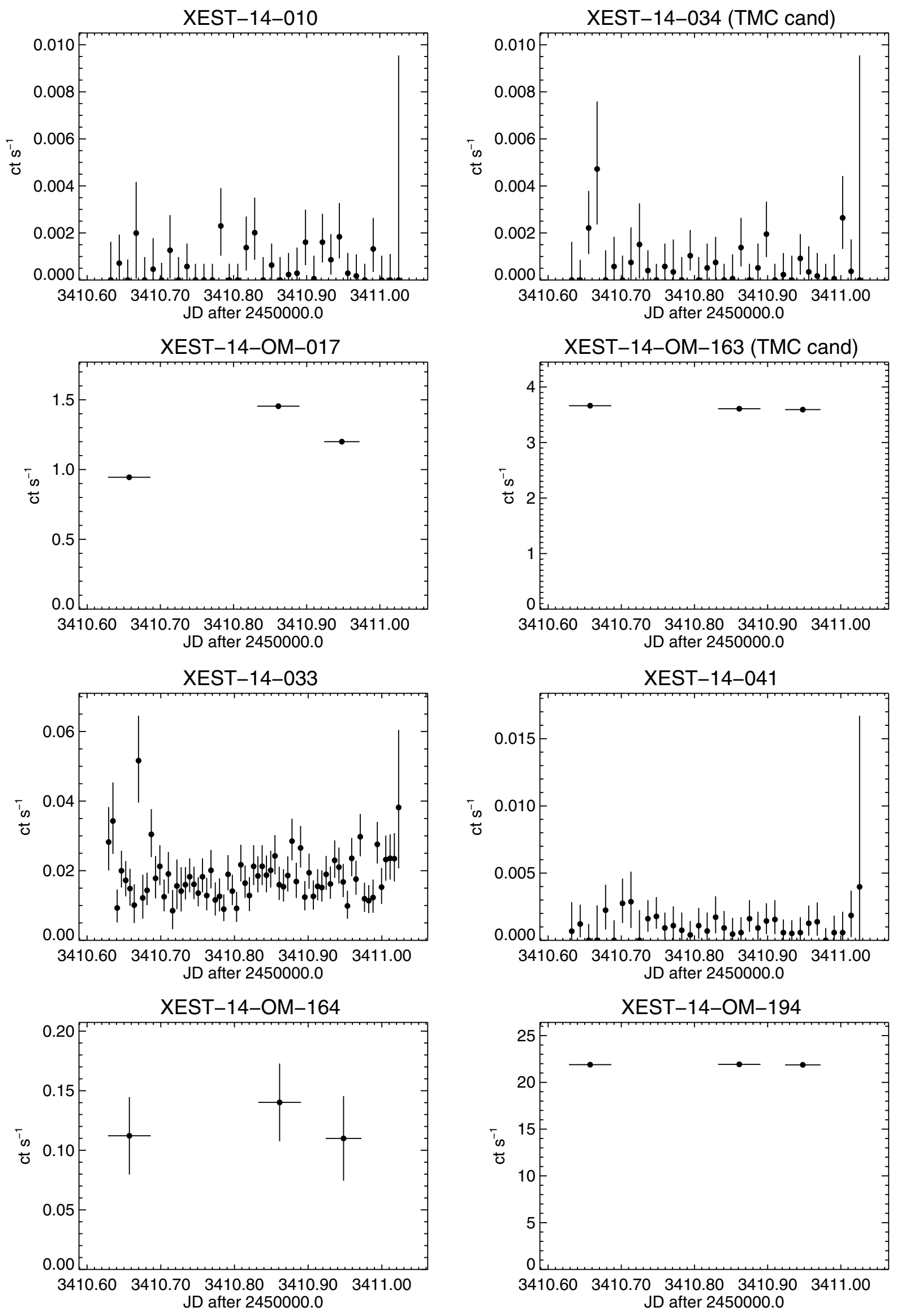

Fig. B.1. continued. 
M. Audard et al.: XMM-Newton OM survey of the TMC, Online Material p 18
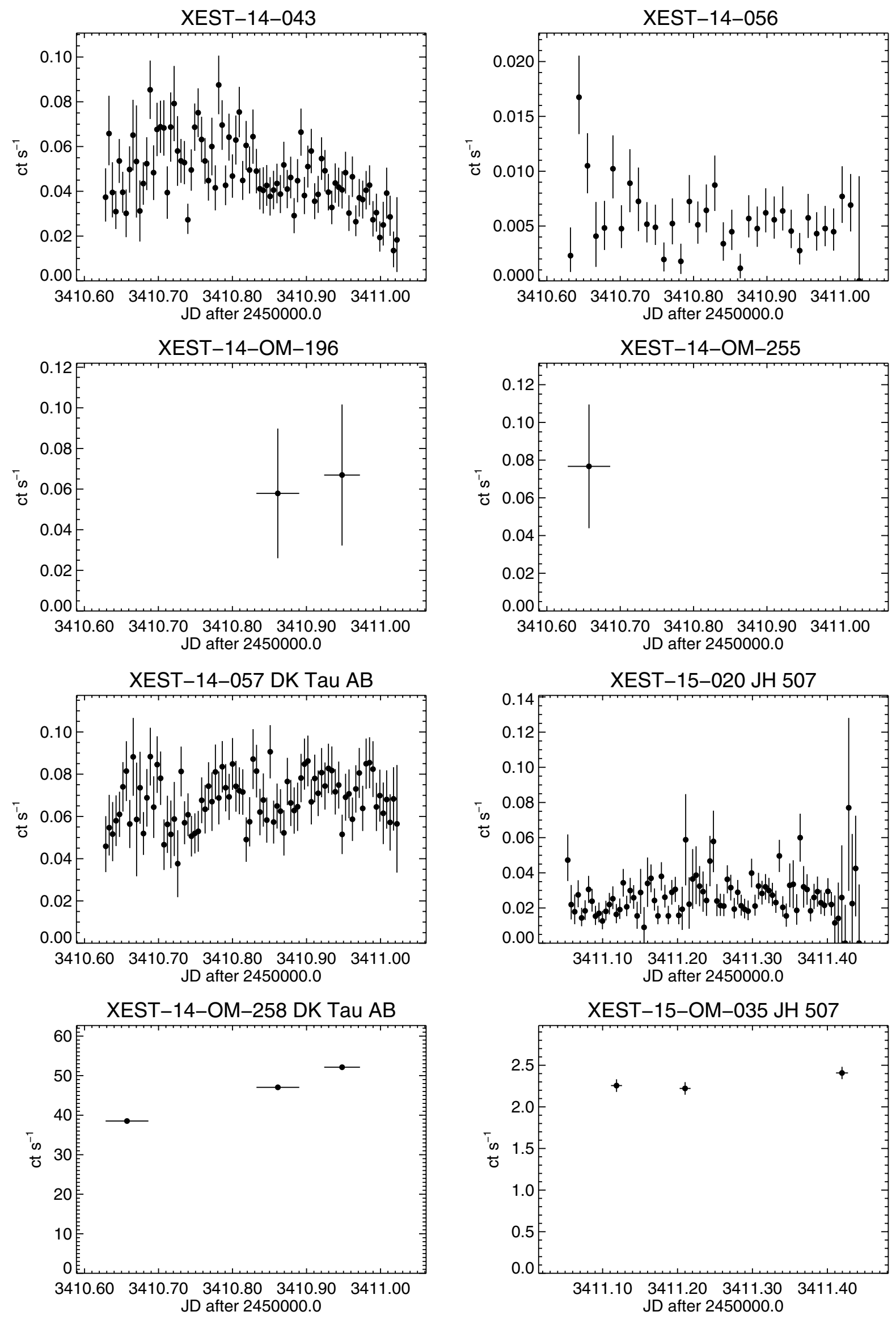

Fig. B.1. continued. 
M. Audard et al.: XMM-Newton OM survey of the TMC, Online Material p 19
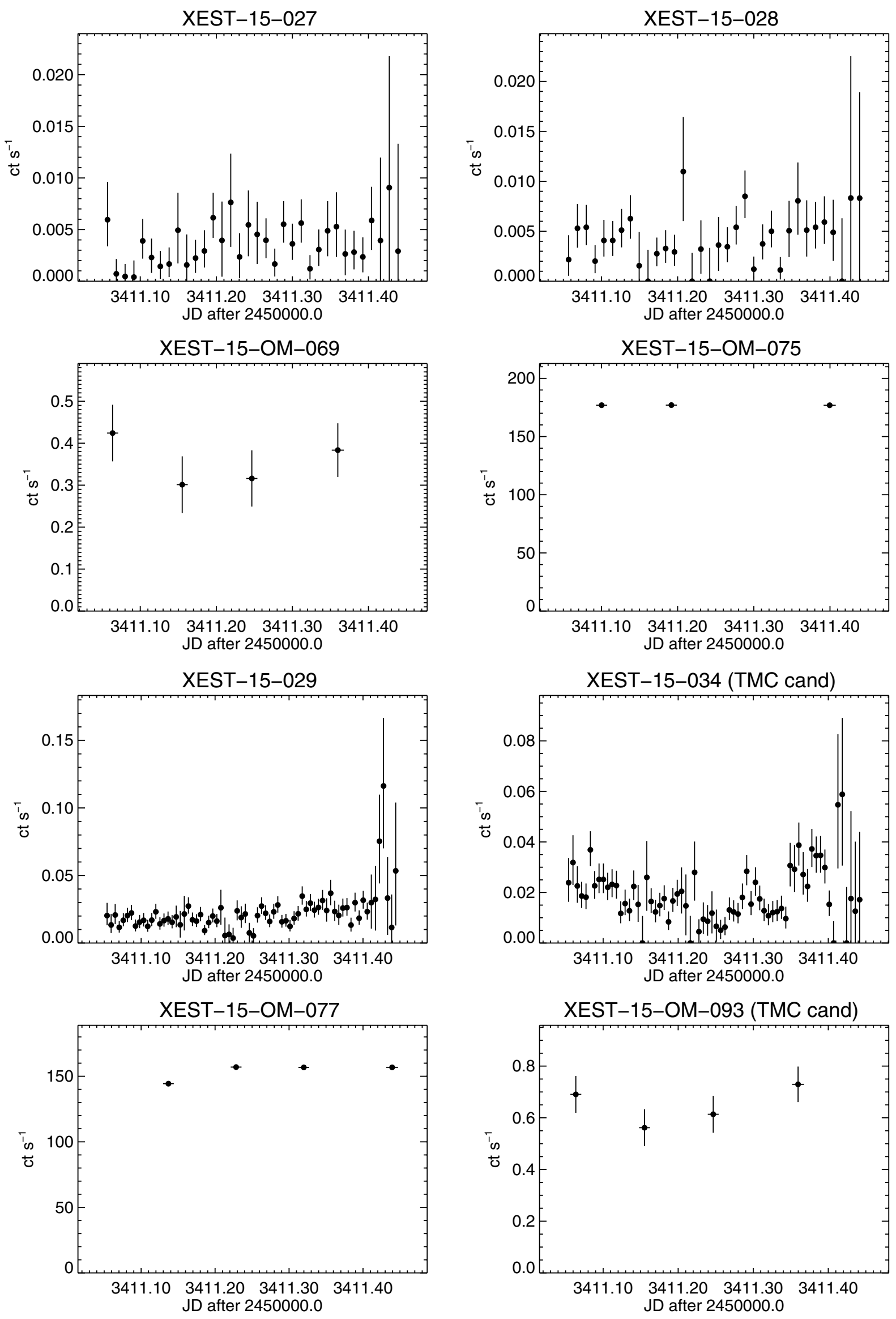

Fig. B.1. continued. 
M. Audard et al.: XMM-Newton OM survey of the TMC, Online Material p 20
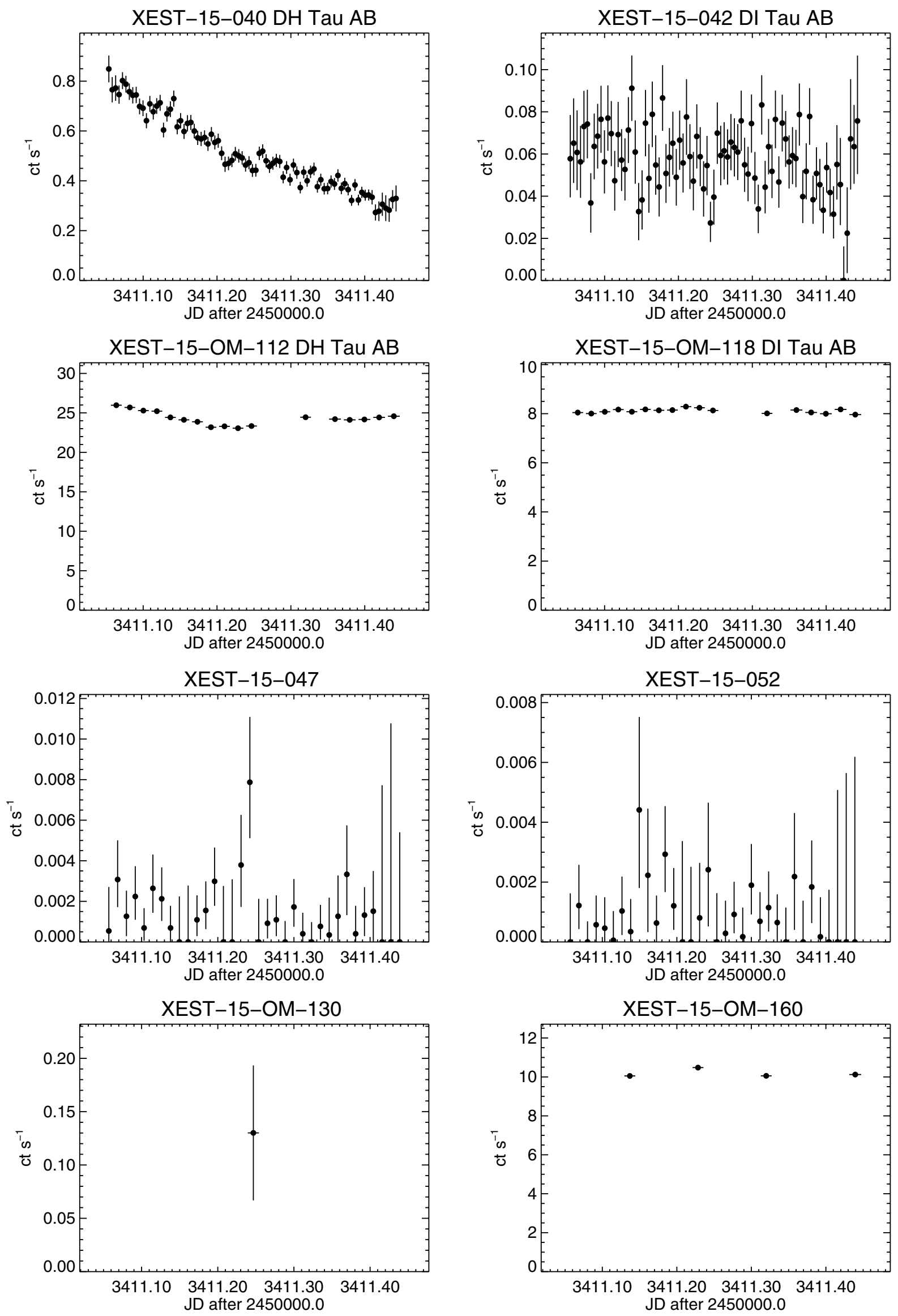

Fig. B.1. continued. 
M. Audard et al.: XMM-Newton OM survey of the TMC, Online Material p 21
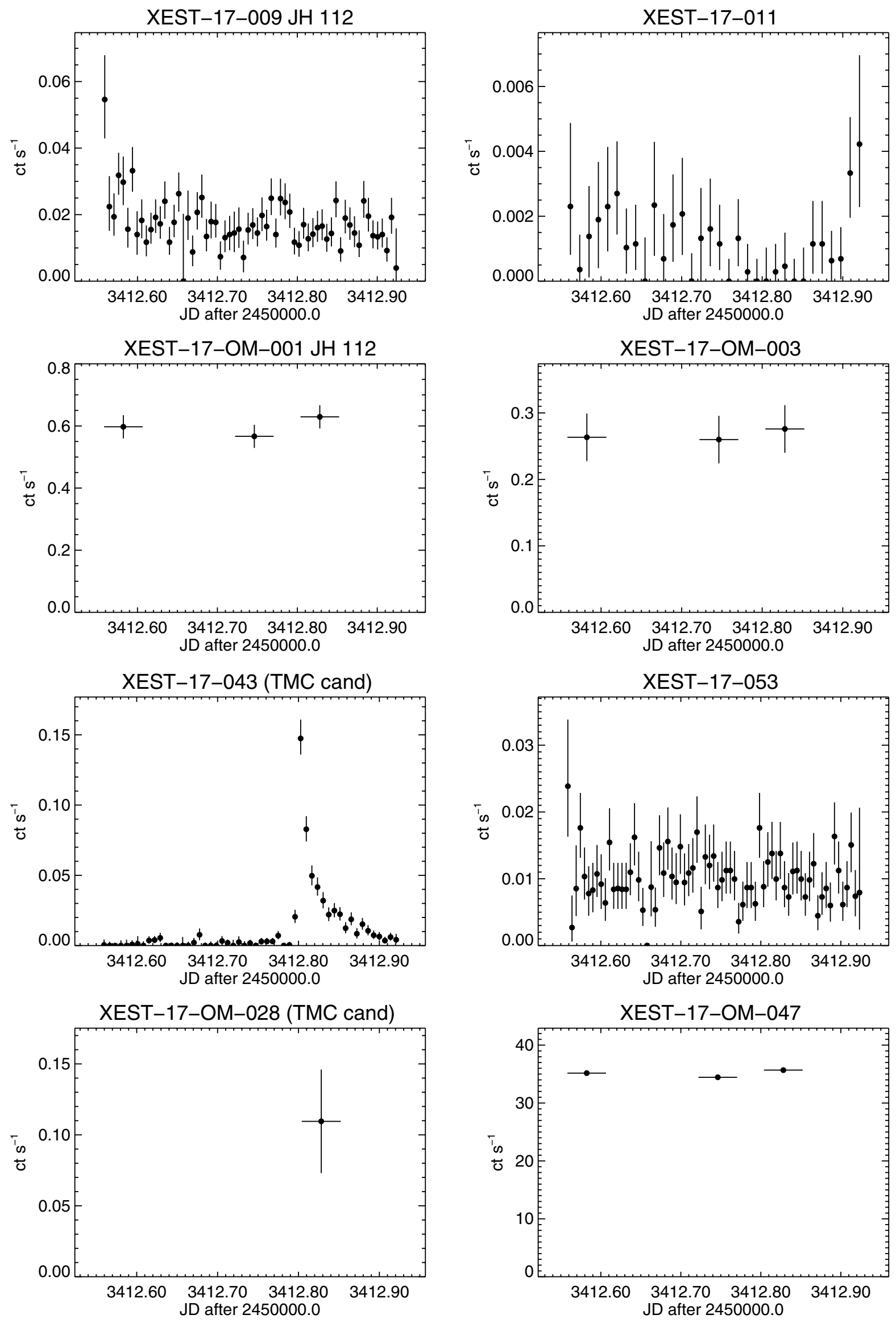

Fig. B.1. continued. 
M. Audard et al.: XMM-Newton OM survey of the TMC, Online Material p 22
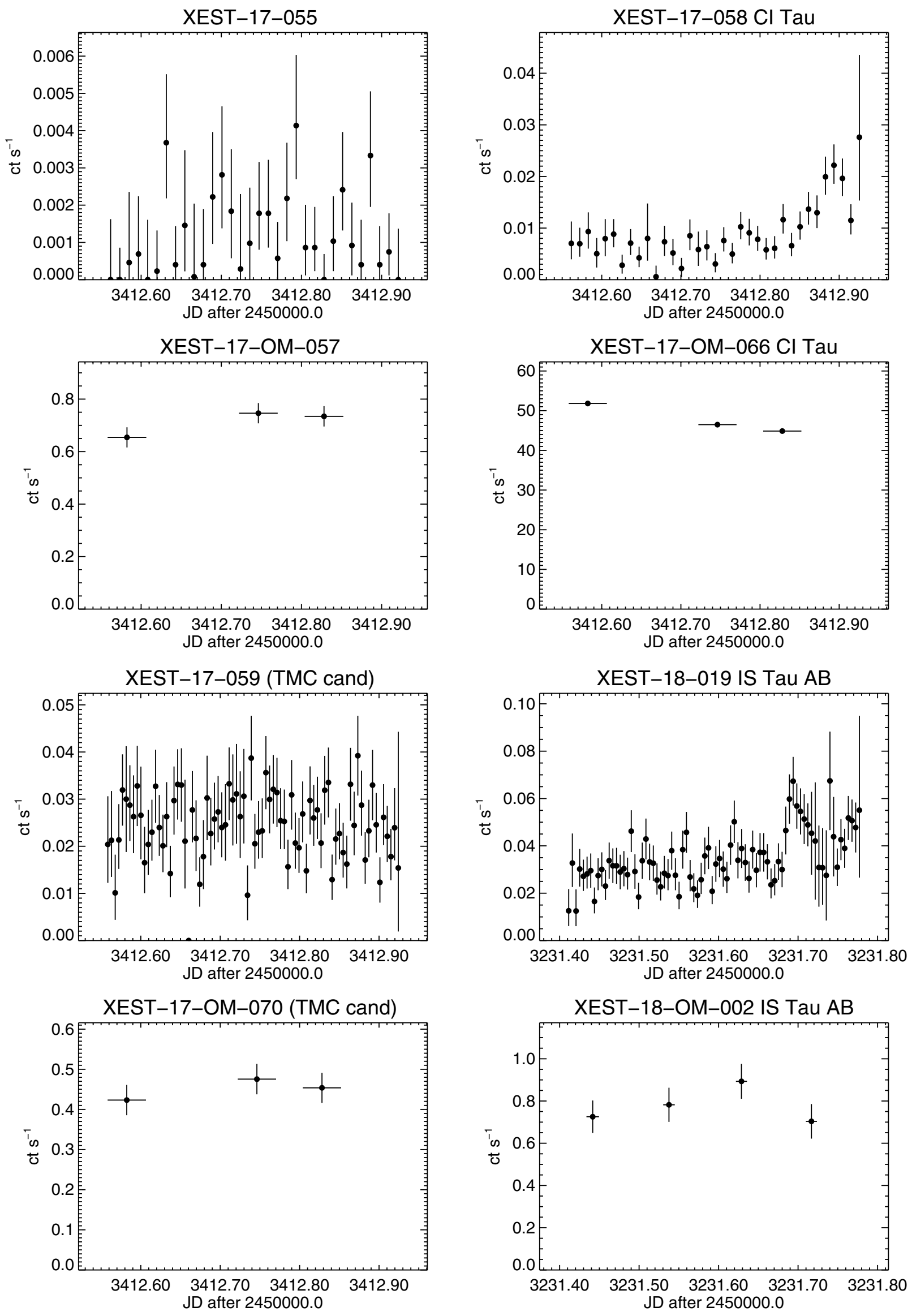

Fig. B.1. continued. 
M. Audard et al.: XMM-Newton OM survey of the TMC, Online Material p 23
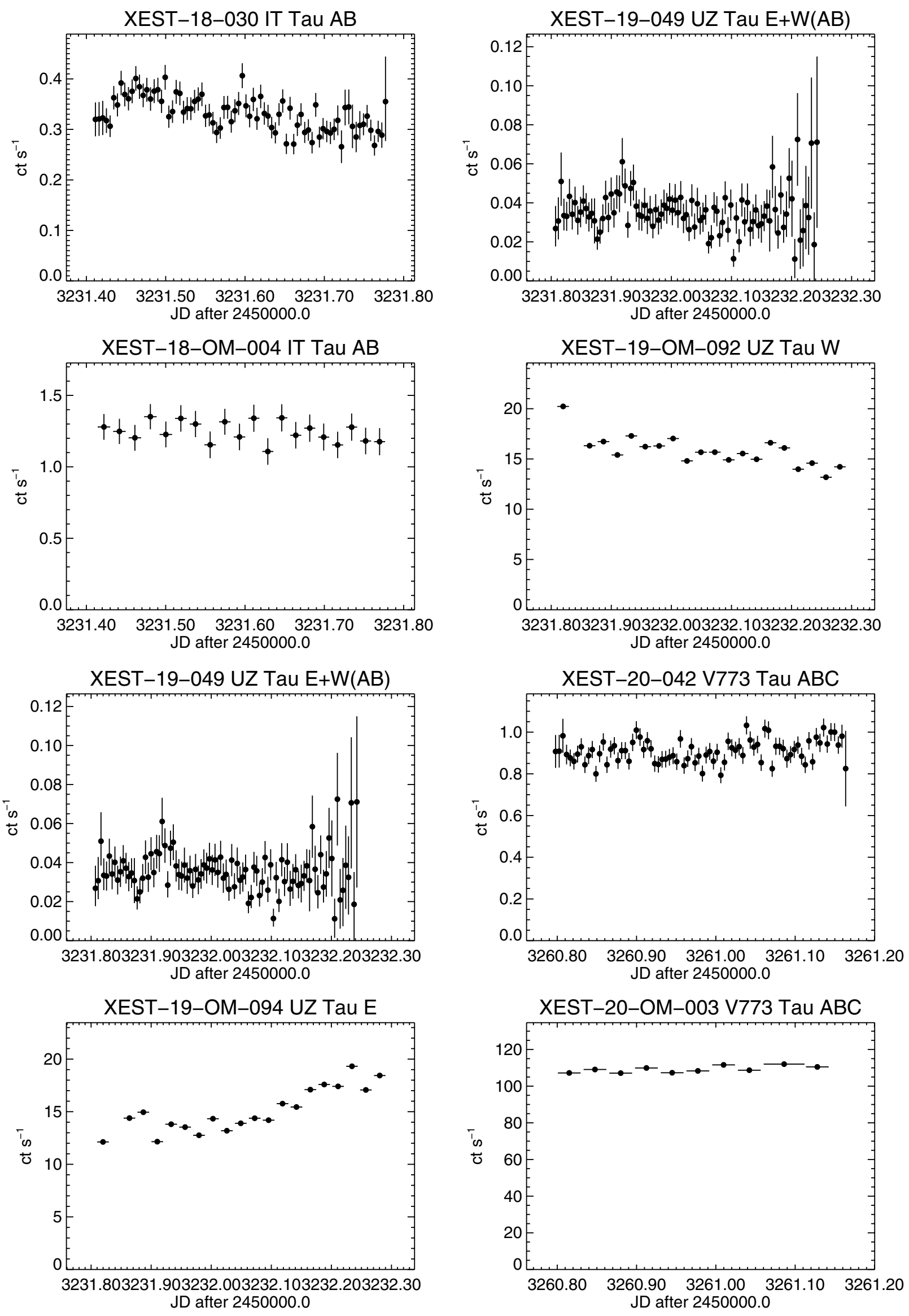

Fig. B.1. continued. 
M. Audard et al.: XMM-Newton OM survey of the TMC, Online Material p 24
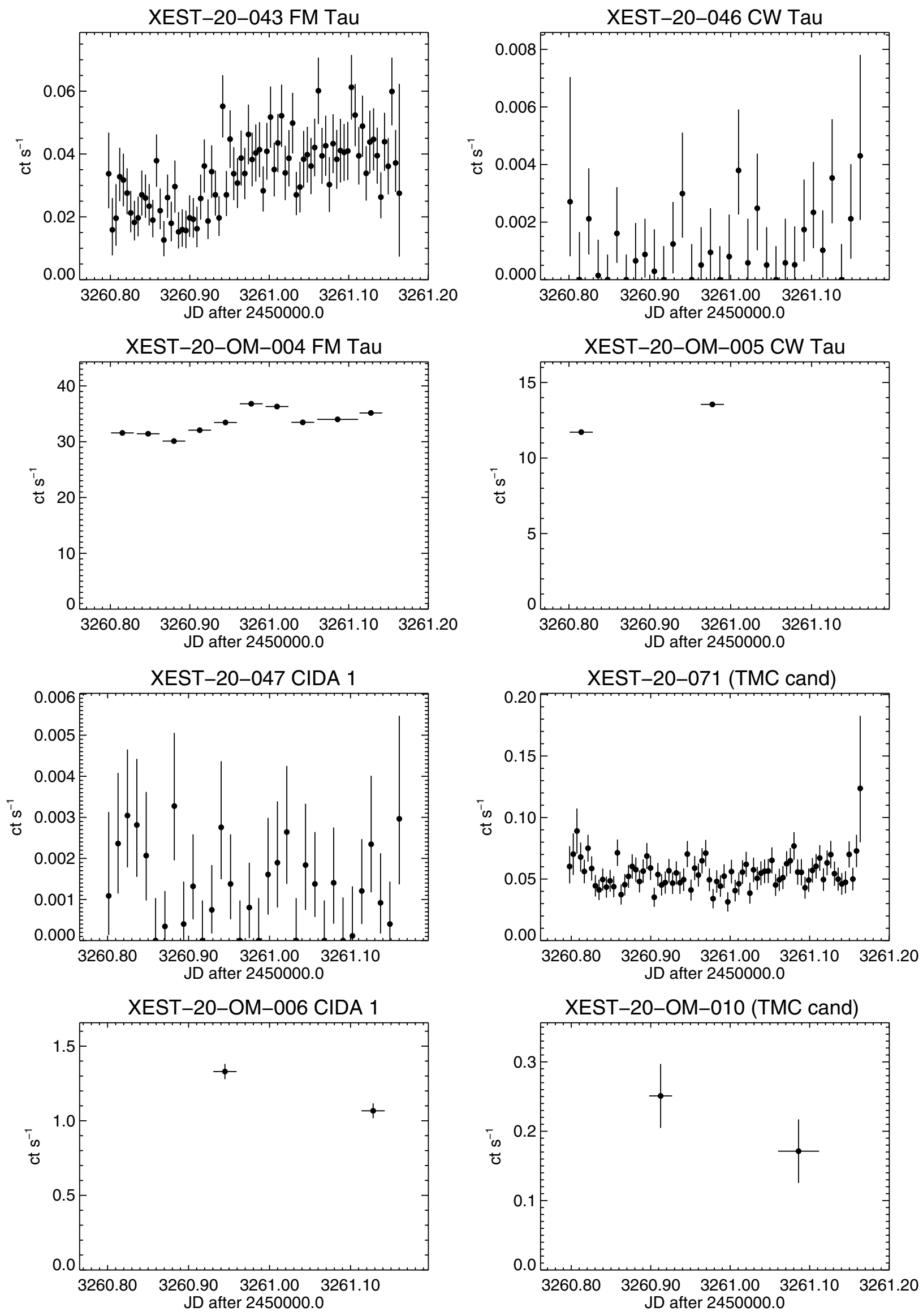

Fig. B.1. continued. 
M. Audard et al.: XMM-Newton OM survey of the TMC, Online Material p 25
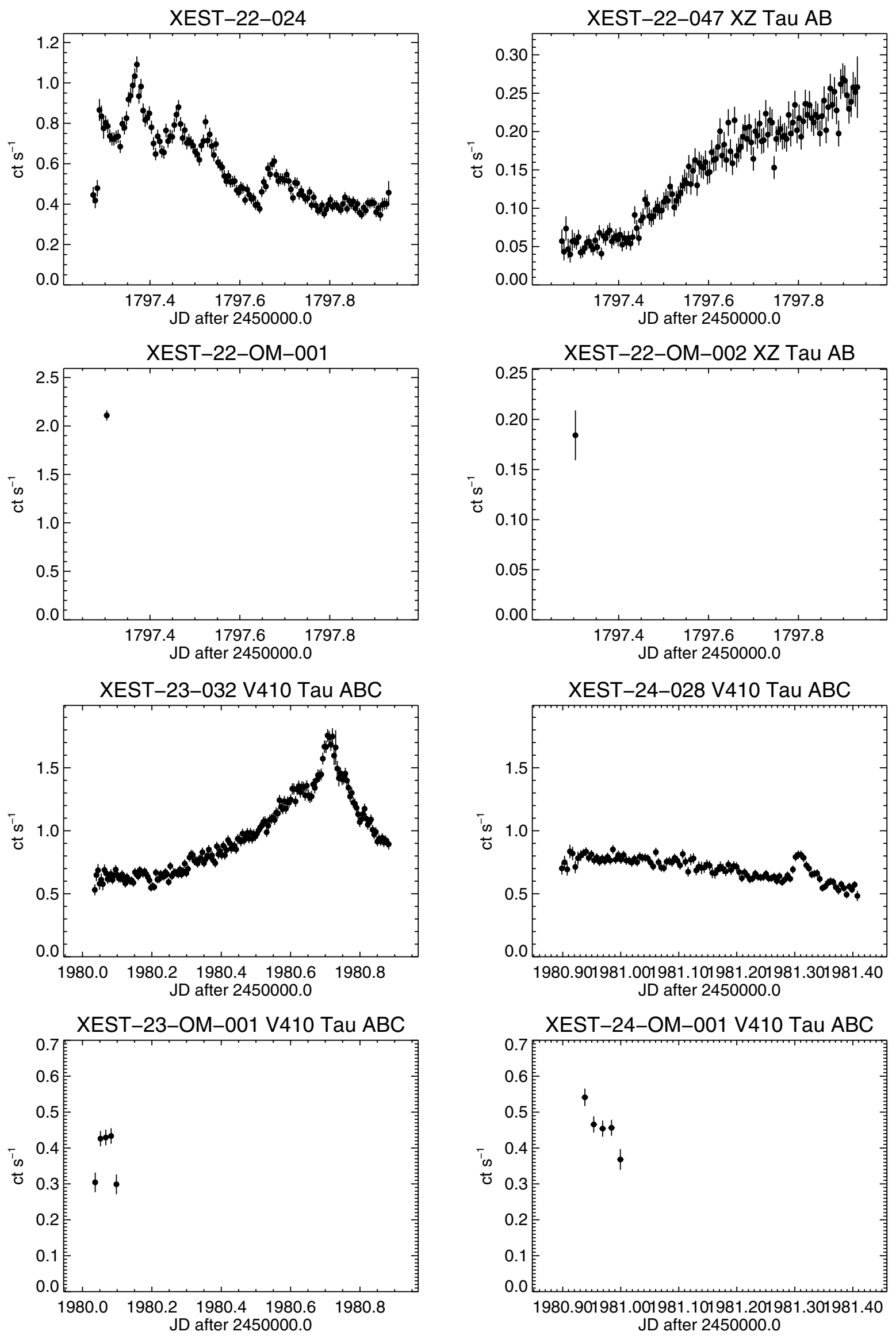

Fig. B.1. continued. 
M. Audard et al.: XMM-Newton OM survey of the TMC, Online Material p 26
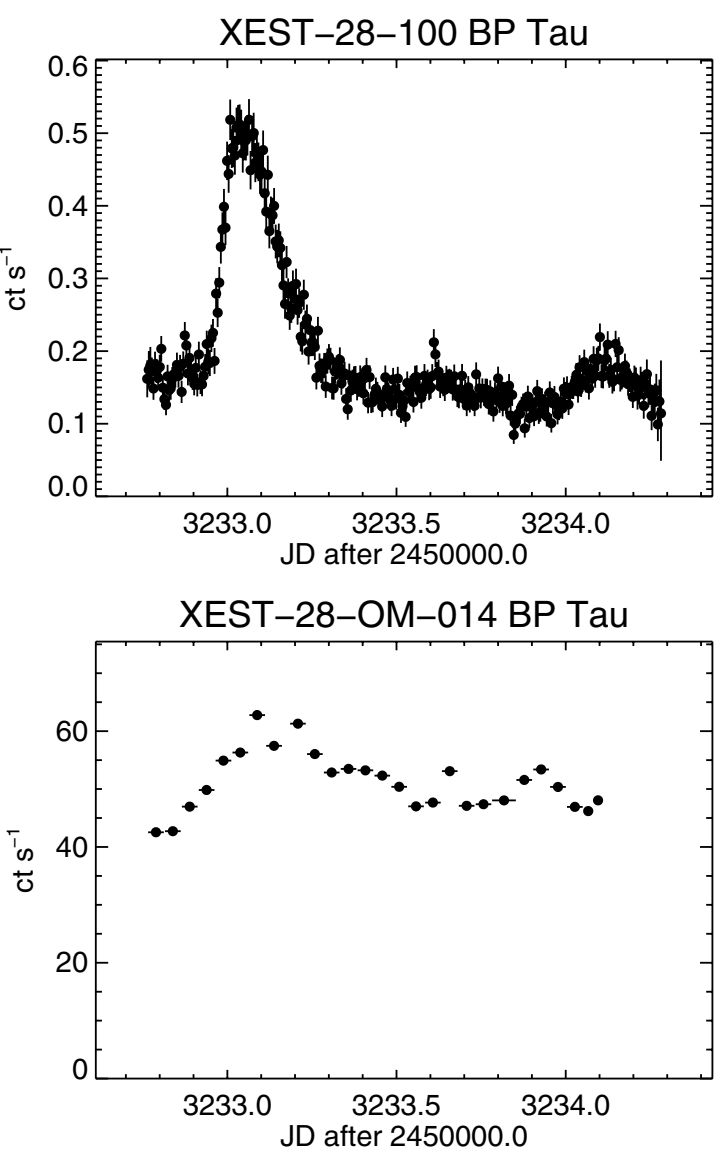

Fig. B.1. continued. 